\title{
MEGALITOS, ESPACIO, PENSAMIENTO
}

\author{
POR
}

\author{
FELIPE CRIADO BOADO (*)
}

RESUMEN Este trabajo, que prolonga un artículo anterior sobre el tema (Criado Boado A), plantea que el megalitismo supone un tipo especial de configuración del espacio social dentro de la cual surgen conceptos específicos de tiempo y de espacio.

En una primera parte se intenta contrastar esta situación con otra distinta que antecedería al megalitismo y que, desde el punto de vista del autor, coincide con la situación que caracteriza a las poblaciones mesolíticas y del neolítico inicial. Se propone que cada una de esas dos situaciones coincide con contextos culturales, marcos sociales y prácticas de subsistencia especificas.

En una segunda parte se particulariza dentro de soluciones megalíticas determinadas con el fin de observar si a través de ellas se expresan diferentes estrategias de ideología y poder funcionando dentro de las sociedades constructoras de los megalitos.

ABSTRACT This work uses ideas of Foucault, Clastres, and Lévi-Strauss to develop an approach to the conceptualization and symbolic use of space and time which legitimized and made possible the construction of megalithic monuments. The megalithic burial pattern is contrasted with those of Mesolithic and earlier Neolithic communities. While the Mesolithic/Neolithic pattern is seen as facilitating the social practice of foragers or shifting agriculturalists, the megalithic ideology would have been developed by settled peasant farmers. Specific types of megalithic monuments are seen as expressing different ideological representations of social power.

Palabras claves Megalitismo, Neolítico, Enterramientos, Espacio, Pensamiento Salvaje, Sociedad Primitiva, campesinos, Ideología, Poder.

\section{AROUEOLOGIA Y MEGALITOS: UN PLANTEAMIENTO BIDIMENSIONAL}

En cierto sentido se podría decir que los Monumentos Megalíticos son una disculpa para pensar. Con ello no pretendemos hacer un acto de reafirmación idealista o de irreverencia histórica. Tan sólo intentamos asumir una perspectiva que nos permita entender qué fueron los megalitos y, sobre todo, hacerlo desde un punto de partida que en vez de considerarse la culminación y superación de las interpretacioness anteriores, se reconozca como un episodio más de una larga cadena de discursos construidos utilizando los Monumentos Megalíticos como pretexto.

(") Departamento de Historia 1. Fac. Xeografía e Histơria. Santiago de Compostela. 
En efecto, decir que los megalitos son una «disculpa para pensan", significa aceptar que, además de cualquier otra dimensión que ellos tengan, esos monumentos son actos, expresiones y concretizaciones de un pensamiento. Ahora bien, en este sentido es preciso reconocer que no sólo fueron el pensamiento de los hombres que los construyeron, sino que además han servido de soporte para creencias y reflexiones de otras sociedades. La propia elucubración arqueológica sobre el fenómeno megalítico, sea más o menos «cientifica» o acertada, es también la expresión de un pensamiento contemporáneo y nuestro que toma forma en torno a los Monumentos Megalíticos.

De este modo se abre un campo bidimensional para el ejercicio de la Arqueología. Creemos que ésta, partiendo de unos mismos principios y planteamientos, y con un mismo aparato teórico (1), deberia dedicarse no sólo a una labor descriptiva que tratase de descubrir a través de los restos materiales de las sociedades arqueológicas los discursos y el discurrir (en el doble sentido del término) de esas sociedades; sino también a una labor crítica que examinase de un modo desmitificador el discurso arqueológico para comprobar en todo momento su racionalidad y relaciones con el contexto en el que se gesta (un esquema de este tipo fue propuesto en Criado Boado 1988a y 1988d).

Dentro de esta segunda dimensión, a la que se aproximan trabajos como los de Fawcett (1986), Leone y otros (1987), Olsen (1986c) o Shanks y Tilley (1987b), se podría ver de qué forma la "producción arqueológica», mientras habla de aquello de lo que parece hablar, en realidad habla de eso otro que intenta negar que habla, con lo que de hecho se convierte en una "proposición ideológica" típica (en el sentido de Althusser 1985: 20), tanto más peligrosa cuanto que esta circunstancia nunca aflora en la conciencia de los arqueólogos y en la superficie del trabajo.

En cambio, si se reconociera, permitiría utilizar esos componentes negativos de forma positiva. Ya que en este caso el trabajo arqueológico seria, en vez de una mera búsqueda en el pasado de sociedades que ya no existen, una indagación dentro de nuestra propia cultura profundizando en ella a través de los discursos que crea para hablar de sí misma mientras insiste en hacer creer que habla de culturas distintas (2).

\section{PERSPECTIVA BASICA: EL ESPACIO «BIDIMENSIONAL» DE LA ARQUEOLOGIA}

Creemos que este posicionamiento teórico-crítico es el más adecuado para abordar el presente trabajo, tal y como intentaremos mostrar a lo largo del mismo y en los dos siguiente subapartados.

\subsection{La «labor crítica" aplicada a interpretaciones previas sobre el Megalitismo}

Partiendo del planteamiento anterior se debe reconocer, por ejemplo, que decir que los Monumentos Megalíticos desempeñan una función social, ya que serían el eje y elemento de equilibrio de una comunidad dada, como por ejemplo propone la interpretación funcionalista de Renfrew (1976a,

(1) Este «aparato teóricow se corresponde en lineas generales con el propuesto por la Theoretical o Critical Archaeology (Hodder 1986 y 1987f, Shanks y Tilley 1987a), y con una línea de pensamiento estructuralista y post-estructuralista (Hodder et all. A, ver también la nota siguiente).

(2) Este planteamiento concreto, así como los recursos teórico-metodológicos en los que se apoya, derivan fundamentalmente de posiciones foucultianas (Foucault 1978a, 1979, 1980b y 1984), y deben mucho a las propuestas que dentro del campo de la Historia ha realizado Bermejo Barrera (1983 y 1987), así como a un tipo de pensamiento post-moderno que, dejando a un lado las connotaciones artificiosas y snobs de este término, entronca con la línea de reflexión madura propuesta por J.- F. Lyotard (1984: 35-41, y 1987: 23-6) o G. Vattimo (1987: 145 y ss.); esta adscripción a la postmodernidad no es una concesión a la moda dominante, sino que surge al valorar qué supone el pensamiento moderno dentro de la Arqueología o en general (sobre este tema en particular véase la nota siguiente - final-). 
1976b, 1983b y 1984b), no sólo supone destacar un aspecto concreto de esas construcciones, sino sobre todo admitir una interpretación de la sociedad como unidad globalizadora que funciona en aras de un equilibrio maximizador, definición que se corresponde bastante bien con el modelo ideal de una Compañía industrial y con el mito básico del capitalismo moderno.

Del mismo modo, señalar que los Monumentos Megalíticos son un símbolo territorial que regula la adaptación al entorno de las comunidades megalíticas (como sugiere el mismo Renfrew y otros autores funcionalistas ecológicos: Darwill 1979, Fleming 1971 y 1973), no significa sólo reconocer un rasgo básico del megalitismo, su componente espacial; sino que además es una declaración realizada desde el contexto particular de una sociedad concreta que ha vivido una "crisis ecológica" (desde 1973 y la crisis del petróleo) que supuso el mayor ejemplo de inadaptación entre una sociedad y su entorno (3).

Finalmente, insistir en que, oponiéndose a lo anterior, los Monumentos Megalíticos son fundamentalmente "expresiones de un sistema de ideología-poden (expresión tomada de Larsson 1985: 107-10 y que desarrollan Tilley 1984, Shanks y Tilley 1982 y 1987a), no es únicamente poner el énfasis necesario en la dimensión olvidada de un fenómeno que es ante todo funerario y, por lo tanto, simbólico. Sino que es ponerlo también en el «econ lógico y natural de un contexto que, después de años de crisis económica, y no habiendo encontrado una solución satisfactoria a la misma, ha situado el estímulo de relanzamiento económico en un consumismo hedonista que precisa insistir en el individuo y revestirlo del narcisismo necesario para convertirlo en el objeto de consumo de la época post-industrial (4). En el seno de esa "post-modernidad" mal entendida y que apenas se podría identificar con post-modernismo (Eagleton 1987), y dentro de un (desmesurado) énfasis en el objeto cotidiano y en el "funcionamiento activo" del mismo para construir el simulacro de la personalidad individual (5), surge una corriente arqueológica que revaloriza la cultura material y convierte a la Arqueología en la disciplina que analiza el funcionamiento de aquella como recurso simbólico para negociar el estatus de cada individuo dentro del grupo (6). Esa Arqueología es la que ahora mira a los Monumentos Megalíticos no como los «símbolos territoriales que queria Renfrew (1987a: 9), sino como «símbolos materiales socialmente activos" (Hodder 1982d).

\subsection{La «labor descriptiva" aplicada al Megalitismo postulados previos y principios teóricos}

Las observaciones anteriores pretenden ser en parte un boceto de lo que una Arqueología crítica puede descubrir sobre sí misma y nuestra sociedad. Pero también intentan señalar el punto de partida del presente trabajo, que pretende describir ciertos elementos del Megalitismo dejados de

(3) Esta interpretación del fenómeno megalítico, así como la enunciada en el párrafo anterior, parten de la teoría fundamental del funcionalismo antropológico (compárense, por ejemplo, sus aspectos «funcionales" y kadaptativos" con las formulaciones de Radcliffe-Brown en la «Introducción» a Estructura y función en la sociedad primitiva, 1986: 9-23); las interpretaciones «espaciales" y «ecológicas» han recibido un espaldarazo a través de la Antropología Ecológica, la más reciente versión del funcionalismo clásico y que, de acuerdo con M. Harris, coronaria el desarrollo de la teoría antropológica (Harris 1978; ver sobre esta estrategia de trabajo Martínez Veiga 1978 y 1985a). En los últimos años se han realizado críticas muy acertadas de las aproximaciones funcionalistas en la Arqueologia en general (Hodder 1982b, Shanks y Tilley 1987a), y respecto al Megalitismo en concreto (Hodder 1984, Midgley 1985). Una crítica del funcionalismo y del ecologicismo antropológico se puede encontrar en Godelier (1976b y 1976a, respectivamente). Pero, yendo más allá de estas críticas localizadas, es preciso sobre todo destacar la tradición intelectual en la que se inscribe toda la teoría funcionalista, valorando su estrecha vinculación con la modernidad y con el orden social que ésta construye, tal y como destaca J.- F. Lyotard (1984: 29-34); estos aspectos están incluso íntimamente relacionados con las formulaciones que la Arqueología funcionalista realiza en torno al megalitismo o a otros fenómenos prehistóricos, tal y como se puede entrever a través de los puntos discutidos en el texto.

(4) Planteamiento que en otros términos se puede encontrar, por ejemplo, en Eagleton (1986: 144-45) o Lyotard (1987: 38).

(5) Es la cultura de "producción de simulacros" descrita y criticada por J. Braudillard (1978: 7), y que se apoya en circunstancias y categorias como las analizadas por J.- F. Lyotard (1987: 45-6) y G. Vattimo (1987).

(6) Esta definición de la Arqueología es la que surge de la obra de Shanks y Tilley (1987a), sin duda uno de los más sugerentes libros arqueológicos de los últimos años, y que recoge una corriente de análisis de la cultura material iniciada a partir del volumen colectivo Symbolic and Structural Archaeology (Hodder 1982a) y que, si bien aporta propuestas válidas, no está exenta de valoraciones críticas como la misma que acábamos de ofrecer. 
lado por otras aproximaciones. Para ello disponemos de una serie de recursos que explicitaremos brevemente a continuación.

A nivel de postulados previos podemos citar tres principales. El primero consiste en reconocer que el megalitismo es un fenómeno de ineludible dimensión espacial. Este planteamiento resulta muy familiar, pues no en vano han insistido en él diferentes trabajos. Ahora bien, beneficiándonos de las aproximaciones consideradas en el apartado anterior, y de un modo un tanto ecléctico, podemos decir que aqui los Monumentos Megalíticos serán entendidos como usímbolos territoriales que son asimismo socialmente activosn.

Con ello nos alejamos de cierto reduccionismo inherente tanto al funcionalismo de la New Archaeology, como a la Structural Archaeology, que se centran respectivamente en los rasgos "territoriales" o «simbólicos" del megalitismo sin preocuparse de establecer la interdependencia entre ambos grupos de elementos. En cambio, una plena comprensión de la dimensión espacial involucrada dentro del fenómeno megalítico supone reconocer que esos monumentos fueron los signos materiales a través de los cuales se expresó la configuración del espacio y del tiempo característica de los grupos megalíticos.

Yendo más allá de ese punto, plantearemos, y éste es nuestro segundo postulado, que el megalitismo expresa un pensamiento especifico, dentro del cual emerge una nueva forma de entender la posición del hombre y la sociedad en el seno de la naturaleza y de pensar el espacio social. Desde un punto de vista convencional se podría decir que el análisis de esta situación constituye el tema central del presente trabajo.

El tercer postulado en que nos basamos consiste en considerar a los monumentos megalíticos, en vez de como «mojones territoriales", «marcas sociales" o "expresiones de una ideología-poder" (Larsson 1985: 107-10), como acontecimientos de pensamiento.

Evidentemente, las dos últimas afirmaciones resultan menos evidentes y son más discutibles que la primera, pues no en vano derivan del planteamiento específico de este trabajo y, por lo tanto se apoyan en principios teóricos que iremos definiendo progresivamente. Por el momento nos limitaremos a deternernos en aquellos que completan los postulados previos y que, al mismo tiempo, nos indican la estrategia de búsqueda más adecuada para profundizar el tema.

De este modo, diremos que el planteamiento del tercer postulado implica, además de otras cuestiones que se tratarán más adelante, utilizar dos perspectivas de observación dispares y complementarias: la primera es una escala general y en ella se examinará el megalitismo desde el pensamiento que lo posibilita; la segunda es una escala específica y en ella se debe considerar cada conjunto megalítico específico, o incluso cada monumento megalítico particular, como un acontecimiento singular del pensamiento anterior. Esta doble perspectiva da lugar a dos niveles de lectura diferentes, cada uno de los cuales constituye uno de los dos apartados principales de este trabajo. A ellos se añadirá un tercer nivel de lectura que es de hecho una valoración global (ap. 4).

Asimismo, y en correspondencia en este caso con el segundo postulado, se debe tener en cuenta que, al igual que antes hablábamos de la bidimensionalidad de la Arqueología, también la labor descriptivo-arqueológica posee una doble dimensión muy clara. Quiere decir ello que, del mismo modo que se admite que una cultura no se reduce a los aspectos físicos y materiales que la caracterizan, sino que sobre todo implica un pensamiento específico, una forma concreta de construir la realidad social (7), se debe reconocer que el registro material de una sociedad no traduce de un modo directo e inmediato lo que ocurre en esa sociedad, sino que refleja un discurso de poder perteneciente a ese grupo y representativo de un determinado sistema de saber-poder (8).

El reconocimiento de esta bidimensionalidad posee particular relevancia para la Arqueología, pues no en vano invalida todo intento de extraer consecuencias de un modo directo y lineal a partir de los restos materiales. Estos, en vez de ser el reflejo directo de la realidad, son el medio a través

(7) Véase M. Godelier (1984).

(8) Estos términos los aplicamos en el sentido de M. Foucault (1978a, 1980a). 
del cual ésta es ocultada, manipulada o construida (9). Pero además de ello adquiere un especial interés dentro de un trabajo como el presente, que pretende observar la dimensión imaginaria de la realidad (10). Por esta razón, cada uno de los dos niveles de lectura que siguen (apartados 2 y 3 ) posee dos subapartados, cada uno de los cuales recoge un término de esa bidimensionalidad.

\section{PERSPECTIVA GENERAL: EL ESPACIO MEGALITICO}

Si se tiene en cuenta que el fenómeno megalítico funde una clara proyección simbólica con una nítida dimensión espacial (11), entonces veremos que la orientación teórica que hemos esquematizado en el apartado anterior (12), a pesar de los problemas que pueda presentar, es muy útil para pensar el megalitismo. Así, mientras es evidente que para describir el primer aspecto es preciso una teoría adecuada a su especificidad, no es menos cierto que para considerar la segunda dimensión es asimismo conveniente mantener esa orientación, ya que, desde nuestro punto de vista, no es posible lograr una aproximación válida y unitaria al espacio sin abordar éste desde una proyección bidimensional que reconozca que el espacio no es sólo un medio concreto o las relaciones del hombre con el mismo, sino también la forma concreta cómo lo entiende cada cultura, o los diferentes grupos de ésta. Así pues, no llega con reconstruir la humanización efectiva (o material) del entorno; también es preciso descubrir la construcción simbólica que lo ciñe; ambas dimensiones reunidas conforman el espacio natural en paisaje social, objetivo hacia el que se deben dirigir los estudios sociales (13).

De este modo, después de habernos fijado en otros estudios en los aspectos materiales del paisaje megalítico (Bello et all. 1987, Criado Boado 1988b y Criado Boado et all. 1986), el presente artículo pretende ofrecer una síntesis, sin duda limitada, de una línea de trabajo que pretendería profundizar en los aspectos imaginarios del megalitismo. Para ello es preciso esbozar los contornos generales del pensamiento dentro del cual se inscribe ese fenómeno cultural.

\subsection{Tiempo, Espacio, Mito, Muerte: del Pensamiento Salvaje al Pensamiento Megalítico}

Para estudiar esta temática, el análisis debe empezar no desde lo material e infraestructural, sino desde el pensamiento y lo imaginario: de acuerdo con la seguda parte de la afirmación que abre el ap. 0 ., y con el planteamiento de los últimos párrafos, es preciso insistir en que los actos, construcciones e invenciones humanas (y los Monumentos Megalíticos entre ellos), antes de ser una forma de llenar el estómagò, un medio de relacionarse con nuestros semejantes, o un recurso para canalizar las tensiones de la sociedad, son un acto de pensamiento y el resultado de una reflexión conceptual. Tal y como se argumentará en adelante, esto debería permitir reconocer que en

(9) Este planteamiento ha sido destacado por los trabajos de la Structural Archaeology (Hodder 1982e: 212, Miller 1982 y 1985); proceden en gran medida de los asertos de A. Giddens sobre la ideología, (ver Giddens 1979: 189-97 y 1984, y también Miller y Tilley 1984b: 13-4).

(10) Utilizamos el término en un sentido semejante al de Duby (1980) y para oponer esta esfera de la experiencia a los aspectos materiales de la realidad.

(11) Esta circunstancia fue reconocida más arriba: se profundizó en ella en Criado Boado (A).

(12) Para una exposición más detallada ver Criado Boado (1988b. 1989, A y B).

(13) Evidentemente no es frecuente encontrar este tipo de aproximaciones dentro de la Arqueologia Espacial, donde dominan en cambio perspectivas funcionalistas, positivistas y cientificistas. En cualquier caso, esta línea de crítica e integración de la Arqueología Espacial convencional dentro de una estrategia teórica más basta, ha sido planteada en Criado Boado (1988b). 
ocasiones la superestructra funciona de hecho como infraestructura, inversión metodológica que puede resultar muy rentable para el estudio (ver también Criado Boado B) (14).

En este punto radica la importancia que dentro de este trabajo posee el concepto pensamiento. Se trata con ello de destacar que esta entidad, en vez de ser un epifenómeno de las condiciones materiales de la sociedad, contiene las condiciones de posibilidad para la construcción social de la realidad que toda cultura realiza. Así pues, y en la medida en que el megalitismo representa la aparición de un tipo nuevo de paisaje adjetivado por unos elementos culturales específicos cuya originalidad respecto a otros "paisajes" culturales radica en que, por primera vez, unas construcciones humanas predominan sobre el espacio permaneciendo además a través del tiempo, debemos suponer que la construcción social del paisaje megalitico se apoya en unos determinados e innovadores conceptos de tiempo y espacio (15).

Tal y como es lícito sospechar, no es fácil para la Arqueología penetrar esa dimensión. Sin embargo poseemos una serie de evidencias generales que nos podrían ayudar. Surgen estas de la rentabilidad explicativa de un modelo interpretativo derivado de sociedades antropológicas actuales, y que ofrece una situación totalmente contrapuesta con la que representa el megalitismo, así como del hecho de que la evidencia arqueológica, megalítica y pre-megalítica, no sólo no contradice ese modelo, sino que incluso lo sostiene.

Este modelo lo aportan las conceptualizaciones de P. Clastres sobre la Sociedad Primitiva (SP), completadas con el trabajo de otros autores (Lizot 1978 y 1980, Gauchet 1980) y con las propuestas de Lévi-Strauss sobre el Pensamiento Salvaje (PS) (16). A continuación, y de modo muy sintético (17), veremos los rasgos que caracterizan a las sociedades primitivas o salvajes.

Su rasgo más universal y característico es la inexistencia en ellas de Estado, entendido éste como un órgano de poder político dividido o separado de la sociedad. Esto no quiere decir que en las SP no exista el poder, sino que es la sociedad en su conjunto quien posee el poder, no habiendo una esfera de lo político independiente a la sociedad misma (Clastres 1976a, Adler 1980). El objetivo del ejercicio de ese poder es mantener alejado el peligro de la división social, con la cual va aparejada una inevitable desigualdad dentro del propio grupo. Es la sociedad contra el Estado (Clastres 1979).

En este sentido, pero también en otros, las SP son "hechos sociales totales», lo que significa que no es posible diferenciar en ellas niveles específicos de actividad social ni, por lo tanto, postular ningún tipo de determinismo entre los diversos sectores de la unidad social (Clastres 1977a: 159) (18).

Esa misma indeterminación se da con la ideología, y ello hasta el punto de que dentro de las SP no se puede aislar un campo de lo ideológico. La religión y el mito constituyen la forma de

(14) Esta inversión, que es también la que M. Godelier opera cuando sostiene que las relaciones de parentesco forman parte de las relaciones de producción dentro de las sociedades primitivas (Godelier 1977a y 1977b), ha sido defendida dentro del campo de la Antropología Estructural por Lévi-Strauss (1972b) y dentro de la Arqueología por Hodder (1986: véase por ejemplo pág. 13).

(15) Sería necesario realizar una discusión pormenorizada del concepto pensamiento, sobre todo de cómo lo entiende LéviStrauss (1964). Unicamente diremos que, en vez de limitarnos a la concepción levistraussina rígida que lo entiende como arazón» (tal y como señala Sperber 1985: 69 y ss.), utilizaremos este concepto dentro de ụna perspectiva más dinámica que no sólo lo conciba como la «sintáxis» de la mente, sino también como el «éxico» con el que una cultura habla, y construye, su realidad social; propuestas en este sentido se encuentran dentro del mismo Lévi-Strauss (1968: 211, y 1986: 181-2); véase Criado Boado 1989 y A.

(16) No es posible identificar el PS linealmente con las SP. Nos proponemos utilizar debajo de ese término únicamente algunas formas y recursos de pensamiento que, además de ser rasgos básicos del PS, son específicos de las SP, y que se refieren fundamentalmente a la conceptualización del tiempo y el espacio dentro de las segundas. Esto no supone olvidar que ni la razón salvaje se limita a las sociedades primitivas, ya que también aparece en nuestra propia vida cotidiana, ni que la sociedad primitiva se limitó a pensar siempre en virtud de una lógica de lo concreto y lo sensible.

(17) Discusión más detenida en Criado Boado 1989 y A.

(18) En palabras de J. Lizot (1978: 74): «Las características generales de estas sociedades han sido puestas en evidencia por Sahlins y Clastres, por no citar más nombres: las posibilidades económicas están bajo la dependencia de las relaciones sociales, de las relaciones de parentesco en particular, las necesidades, de número limitado, pueden ser satisfechas fácilmente por un trabajo razonable y el trabajo productivo se interrumpe una vez que están completas, la autarquía es el ideal del grupo doméstico, el cual constituye la unidad fundamental de producción, las relaciones de poder son inexistentes o reducidas». 
interrelación entre la sociedad y el poder que la SP ejerce sobre si misma para civitar la división (Clastres 1977a: 159). El discurso de estas sociedades establece un orden. Y ese orden mantiene a la sociedad igual a sí misma. Pero este orden no proviene de seres humanos, pues si asi fuera no sería respetado, sino que procede de un discurso-dado por los Dioses a los Ancestros. No existe por lo tanto una ideologia manipuladora o subordinada a un poder de dominación, va que "la naturaleza de las SP excluye la posibilidad de ese discurson (Clastres 1978: 173), sino que la ideologia es la condición básica de pervivencia y preservación de las SP: «en vez de actuar como 'superestructura', es inherente al ser social primitivom (Clastres 1977a: 159).

Este discurso transmite significados y utiliza recursos muy concretos. Entre todos ellos nos interesa destacar ahora la conceptualización del espacio y del tiempo que aparecen en su seno.

Respecto a lo primero, se debe resaltar que dentro de las SP existe una plena correspondencia entre el tipo de utilización que se hace del espacio, y la simbolización que se efectúa del mismo. Abundantes testimonios etnográficos señalan el profundo conocimiento que estas sociedades poseen de la naturaleza, rasgo que se documenta entre cazadores, recolectores o agricultores "primitivos" (Clastres 1986, Lévi-Strauss 1963, Maestre Alfonso 1978: 78 y ss., Service 1973: 78-81), y que genera un comportamiento profundamente *ecológico" por parte de estos grupos plasmado en la adopción de formas económicas muy armoniosas con el medio.

Pero este conocimiento no se limita sólo a satisfacer necesidades, sino que sobre todo "corresponde a exigencias intelectuales" (Lévi-Strauss 1964: 24). Sirve para pensar. Revela con ello una profunda relación entre la naturaleza y la cultura, conexión que se utiliza para, fundándose en analogías naturales, ordenar la cultura, ordenar la naturaleza y, en definitiva, ordenar la realidad social. Tal es la función del "operador mítico" (Lévi-Strauss 1965), de los sitemas de clasificación del "pensamiento salvajé" (Lévi-Strauss 1964), o la que aparece en el "pensamiento mítico" (Lévi-Strauss 1976: 611-4), efectuándose de esta forma una dilución de la naturaleza en la cultura y de la cultura en la naturaleza (Lévi-Strauss 1976: 137-8).

El análisis de la conceptualización del tiempo en las SP muestra asimismo una estrecha correspondencia con su categorización y posición respecto al espacio. La mayor parte de los testimonios que se pueden reunir, verifican la ausencia de unas nociones temporales semejantes a las nuestras y construidas sobre un eje de prolongación-sustitución (por ejemplo, Service 1973: 82). Lo que derivaría de un intento consciente por parte de las SP de negar el efecto de la experiencia del tiempo, de rechazar el impacto del cambio y del dis-currir temporal y de volverse en definitiva contra toda dinámica que pueda alterar su ser social primitivo (Lévi-Strauss 1973: XLIV-XLV). Negar el tiempo es una forma de afirmar la permanencia de la sociedad (Clastres 1981b). De ahí la inexistencia de sistemas de cómputo del tiempo o de sistemas para «introducir un mínimo de orden cronológico en el pasado recienten (Clastres 1986: 240).

Pero tal vez sean el mito y la muerte los campos más adecuados para observar la posición de las SP hacia el tiempo. Clastres indica que en las SP de América del Sur hay una clara distinción entre dos tipos de muertos: los muertos antiguos o Ancestros y los muertos recientes o Antepasados (Clastres 1981b). La sociedad de los vivos reconoce una estrecha proximidad con los ancestros, muertos pasados que nadie recuerda y que se entremezclan con divinidades y héroes del grupo. Estos ancestros ocupan el tiempo del mito, que es a su vez el tiempo, aunque tal vez fuera mejor decir «lugan, del cual proceden las reglas de la sociedad, su discurso mítico y la sociedad misma. Por ello la referencia al pasado mítico y a los ancestros es siempre positiva. Dado que éstos fueron quienes fundaron la sociedad y le dieron un conjunto de reglas a través de sus mitos, constituyen la condición de supervivencia de aquella.

La relación con los ancestros opera una re-aplicación continua del pasado mítico en el presente, lo que niega el flujo del tiempo y destaca la continuidad cultural. De este modo, la perseverancia que la SP persigue, encuentra su expresión práctica en la negación particular del tiempo y dis-currir cotidiano que las SP atestigüan en su vida diaria, en la misma ausencia, ya no de sistemas de calendario, sino incluso de la más mínima preocupación por el tiempo, por el pasado y por el futuro. 
En cambio la referencia a los muertos recientes, a los antepasados, es siempre negativa, pues no en vano son la mejor expresión de los peligros del tiempo. Ellos, en vez de consolidar la continuidad de la sociedad, recuerdan continuamente que el tiempo fluye, y demuestran que éste puede destruir y afectar la identidad cultural (ver p. ej. Harris 1986: 19).

De este modo, la relación entre la sociedad viva y los antepasados, deviene en una relación de alejamiento y ocultación. Todos los ritos acerca de la muerte pretender hacer olvidar su efecto. La inexistencia de rituales funerarios elaborados, la ausencia de culto, de ceremonialismo funerario y de cementerios, son otras tantas estrategias para conseguir ese objetivo. Los muertos poco menos que se abandonan sin apenas enterrarlos (Clastres 1981). Se dejan en canoas en los ríos, en plataformas o en lo alto de los árboles, esperando que el tiempo borre sus restos. Otras veces se los come (Clastres 1986: 220 y ss.). O se entierran en necrópolis alejadas de las áreas de actividad normal del grupo, en lo más profundo de la espesura, y con la norma estricta de no poder ser visitados por los vivos (Carmichael et all. 1985). Jenness (1955), en una revisión de las tribus indias de América del Norte verifica que entre todos los grupos cazadores-recolectores y entre agricultores de rozas, las estrategias funerarias son siempre de esos tipos.

Podriamos añadir más datos y descripciones sobre las sociedades primitivas y salvajes. Esto sería muy rentable para nuestro trabajo. Pero, en beneficio de la brevedad, únicamente propondremos que, sobre este fondo del espacio-tiempo primitivo, del paisaje salvaje, se destacan con mayor nitidez y con rasgos totalmente opuestos los monumentos megalíticos. Tal y como apuntamos más arriba, y de acuerdo con el planteamiento que hemos asumido, una transformación de este estilo sólo se puede dar a través de un reajuste pertinente y previo del aparato conceptual con el que la sociedad piensa la realidad.

\subsection{La Construcción Megalítica del Paisaje: del Paisaje Salvaje al Paisaje Campesino}

La profunda transformación o fisura que señala el megalitismo se puede concretar en cinco puntos.

Dos de ellos se refieren a la proyección y conceptualización espacial que el fenómeno megalítico representa. Así, en primer lugar destaca el hecho de que los megalitos sean monumentos que predominan sobre el entorno, y en segundo lugar la circunstancia de que, a través de ello, supongan la imposición de un efecto humano permanente sobre el espacio, creando paisaje humanizado.

Otros dos se relacionan con la dimensión temporal y funeraria del megalitismo. En este sentido se debe resaltar ante todo el que sean construcciones que desafían al tiempo y realizadas con una clara vocación de permanencia. Pero no es menos importante el que sean obras monumentales cuya misión primaria es exhibir la muerte, hacerla visible.

Un quinto punto seria el hecho de que el megalitismo supone una expropiación de trabajo. Mientras los cuatro aspectos anteriores atestigüan los cambios en el mundo conceptual que subyacen (y posibilitan) al megalitismo, este último punto posee una clara significación sociológica, ya que evidencia los cambios ocurridos en el entramado social, y verifican que la sociedad megalítica posee un poder dividido o en vías de división, pues "sólo a la fuerza el hombre trabaja más allá de la satisfacción de sus necesidades" (Clastres 1979, ver sobre ello Criado Boado et all. 1986: 172 y ss.).

Ahora bien, las tres primeras características señaladas no son exclusivas del megalitismo, sino que definen también cualquier tipo de fenómeno monumental. De aquí se pueden derivar dos observaciones.

En primer lugar, sería posible esperar que eso que impropiamente denominamos "pensamiento megalítico" sea un fenómeno mucho más complejo y basto que el megalitismo estricto. En realidad deberíamos hablar de un tipo de orden cultural y social dentro del cual se legitima la construcción de grandes monumentos, y que a escala global aparece como algo totalmente opuesto al orden 
primitivo-salvaje. Evidentemente la validez de esta propuesta sólo puede ser de alcance general, y nunca particular, pues dentro de ese orden aparecen de hecho construccione's tan dispares como los megalitos atlánticos, los woodhenges ingleses, los long barrows norteeuropeos o, incluso, las long houses danubianas o los telles del neolítico centroeuropeo. Todos ellos formarian un gigantesco grupo de transformaciones, cada una de las cuales debe ser explicada en su contexto, pero que todas juntas participan de un orden cultural (conceptual) común.

En segundo lugar, la anotación anterior nos permite ofrecer una alternativa optimista a uno de los problemas fundamentales que presenta el megalitismo. Sabido es que en todo el ámbito de dispersión del megalitismo atlántico los monumentos funerarios son tan abundantes como escasas las evidencias de habitats. Esto ha dado pie a todo tipo de especulaciones sobre el carácter nómada y pastor de las comunidades megalíticas. Sin embargo, observando esta situación desde la perspectiva del ugrupo de transformaciones" que acabamos de señalar, habría que plantearse si acaso la invisibilidad de los espacios de los vivos, no es la consecuencia lógica de la visibilidad de los espacios de los muertos. Y del mismo modo, tampoco sería arbitrario, sino el par opuesto de esta situación, el hecho de que en todas las zonas europeas en las que aparecen grandes y monumentales poblados (dejando al margen el conjunto de asentamientos proto-urbanos con necrópolis megalíticas que plantean una problemática independiente que no entramos a considerar en este artículo), falten en cambio, ya no megalitos, sino incluso tumbas visibles o de cierto carácter monumental. Todo es como si el predominio de la sociedad sobre el medio y la continuidad de ésta en el espacio y en el tiempo, se pudiera expresar de dos formas distintas y excluyentes: en un caso a través de los muertos y en otros a través de los vivos (19).

Frente a estas observaciones de carácter general, lo que contribuye ante todo a particularizar al megalitismo es el cuarto rasgo enunciado más arriba, esto es, el hecho de ser un monumentalismo para exhibir la muerte.

A continuación, y sin pretender plantear ninguna nueva teoría sobre los orígenes de la tradición megalítica, intentaremos definir algunas de las coordenadas sobre las que se inscribe este fenómeno, esa fisura del orden salvaje. Para ello nos centraremos en dos puntos: por un lado en el examen del contexto y marco en el que aparecen las primeras construcciones monumentales, y por otro en la valoración del contraste que el monumentalismo, megalítico o de otro tipo, presenta respecto a la situación Mesolítica y de la primera parte del Neolítico.

Este segundo punto precisa reconocer que el complejo mesoneolítico, en contra de las opiniones tradicionales que señalarian una profunda discontinuidad entre ambos períodos, forma parte de una misma unidad que se puede identificar con la sociedad primitiva y el pensamiento salvaje.

Esto se verifica, a nivel del espacio y de prácticas de subsistencia, no sólo en los datos arqueológicos recientes que señalan la escasa distancia entre Mesolítico y Neolítico (por ejemplo: Burenhult 1984, Ford 1979), sino sobre todo en el hecho de que el Neolítico debe ser entendido, en vez de como una fase de revoluciones y de inicios de nuevas formas de explotación del medio, como una etapa que mantiene en sus rasgos básicos el tipo de interacción con el entorno que se percibía ya en los grupos cazadores-recolectores (Cohen 1981: 32 y ss.).

Este tipo de interacción se percibe en las actividades complejas que implican un tratamiento sofisticado de las plantas y animales, y que aparecen ya en ciertos grupos mesolíticos y que apenas se diferencian de formas primitivas de agricultura o ganadería. Estas actividades son denominadas gardening en la bibliografía especializada, connotando con ello una práctica a medio camino entre la recolección, la «jardinería” y la agricultura (Larsson 1985, Sherrat 1980).

En todas estas prácticas de subsistencia se percibe que la primera condición para la supervivencia de la sociedad es mantener la naturaleza dentro de su orden natural correcto, y sin ocasionar efectos importantes sobre ésta (20):

(19) Hodder (1984) y Midgley (1985) apuntan a ciertas consideraciones en este sentido. Sin embargo, mientras la perspectiva funcionalista del segundo no le permite más que hacer una versión de la visión de Renfrew de los monumentos como símbolos sociales aplicada a las long houses, y en la que se pierde toda consideración de tipo simbólico, la estrategia del primero se extravía en viricuetos demasiado particularistas y subjetivos.

(20) De hecho los primeros cambios ocasionados sobre el medio fueron de tipo "conservacionista*, véase por ejemplo: Cohen 1981: 32 y ss. y Day 1953. Los mismos «clareos" o incendios de época mesolítica habrian estado presididos por esta lógica (Jones 1976, Mellars 1975, Simmons 1979). 
Todo ello debería contribuir a imponer la idea de que el Neolitico, en vez de ser una domesticación de la naturaleza, es la naturalización de la cultura (21). En este sentido respondería plenamente a la lógica espacial del entramado primitivo-salvaje antes descrito. Dentro de ese entramado, y a pesar como señala P. Clastres de la similitud formal entre todas las SP en otros aspectos, el repertorio de bases de subsistencia posibles es tremendamente amplio y su elección por una sociedad u otra totalmente indeterminista (Clastres 1976b, Sahlins 1977). De hecho, ese entramado es un continuum a lo largo del cual una misma sociedad puede adoptar y abandonar la agricultura o una subsistencia cazadora según le venga en gana (Clastres 1981a, Lévi-Strauss 1963).

La misma imagen de continuidad surge si observamos lo que se refiere al tiempo y los ritos funerarios. En efecto, en virtud de lo que se dijo sobre este tema en el apartado anterior, tal vez sea necesario empezar a considerar como una evidencia significativa la ausencia de evidencia sobre enterramientos en la mayor parte de los periodos prehistóricos pre-megalíticos. Esta falta, en vez de ser debida al azar o a un problema de documentación se debería fundamentalmente a una estrategia consciente por parte de los grupos prehistóricos de ocultar la muerte.

Por otro lado, la mayor parte de los enterramientos que poseemos de momentos paleolíticos, epipaleolíticos e incluso del neolítico antiguo, se corresponden bien en su mayor parte con ese tipo de prácticas de ocultación (May 1986): deposición de cadáveres entre basuras y escombros, (como és el caso de Los Azules o, en grado máximo, el enterramiento en concheros), desmembración de los cuerpos, canibalismo ritual...

En contraposición con la situación anterior, la especificidad del megalitismo viene dada no sólo por los rasgos que lo definen, sino también por aparecer sobre un determinado marco económicosocial y en el seno de un contexto cultural nuevo.

En lo que se refiere a la relación con el espacio, los comienzos de la arquitectura monumental sobreimpuesta al paisaje coinciden con el inicio de la ocupación y transformación del entorno a gran escala. Antes de niveles del Neolítico Medio, las huellas del efecto humano sobre el medio son escasas y ambigüas. Pero a partir de este momento serán ya inequívocas. Por todas partes se invierte el tipo de relación cultura-naturaleza que caracterizaba el orden primitivo-salvaje. El paisaje social, en vez de ser el configurado por la naturaleza y ocupado por el hombre, contribuyendo así a la "naturalización de la cultura", empieza a ser un paisaje creado en todos sus rasgos por el hombre. Como resaltando una profunda coherencia entre el efecto ambiental de las prácticas de subsistencia, y la ocupación simbólica de ese ambiente, aparecen los monumentos imponiendo un orden humano sobre el medio y configurando un nuevo tipo de paisaje.

Ahora bien, esta fisura no ocurre sin más. Sino que, según nuestra opinión, concuerda con la aparición de un nuevo modo de vida que señala una profunda discontinuidad con el orden mesoneolítico: el modo de vida campesino.

Este se impone a partir del Neolítico Medio (o Calcolítico, según las zonas) a través de la revolución neolitica de los productos secundarios (Sherrat 1980). Supone el inicio de la settled agriculture (ver sobre ella Boserup 1965), es decir de la agricultura en comunión con el terrazgo, amparada por prácticas estables: barbecho, abonado arado, ganadería estabulada, iracción animal. Hay una fisura mayor entre el campesino y el agricultor de rozas del Neolítico inicial, que entre éste y el cazador-recolector. En este sentido es significativo el que el megalitismo siempre aparezca en momentos desarrollados del Neolítico y sobre una base neolítica previa (Jorge 1981).

El campesino representa el inicio de una auténtica domesticación del espacio, entendido ello como la imposición a éste de un orden cultural. El campesino es el hombre que llega a ser esclavo de la naturaleza, que sufre los riesgos de los accidentes naturales, y por ello utiliza su cultura para domar la tierra, para dominar la naturaleza. Con el campesino aparece la producción, la obtención de excedentes y desaparece la jornada laboral de tres horas de la que habla Sahlins (1977). El campesino termina, en suma, requiriendo el control más pleno posible del territorio. Su pensamiento,

(21) Ver sobre este tema Criado Boado (1989 y A). 
en vez de legitimarse a través de la naturaleza, concibe a esta como un enemigo y le impone un orden a través de él.

En este sentido es muy significativa la aparición en algunas zonas de sistemas de campos cercados relacionados con monumentos megaliticos (Caulfield 1978, 1983 × 1986). La posibilidad de limitar el territorio, de expropiarlo, era ajena al pensamiento primitivo. Esta práctica se va a generalizar a partir del Neolítico final y en la Edad del Bronce (Bowen et all. 1978). Pero la preminencia de las construcciones monumentales sobre el paisaje, señala ya un primer tipo de apropiación del territorio que, por el momento, se apoya fundamentalmente en recursos simbólicos. La visibilidad del monumento, sea funerario o de otro estilo, sea incluso un poblado permanente, representa la reivindicación del territorio.

A partir de aquí es posible pensar los megalitos como símbolos territoriales, y superar las limitaciones y simplificaciones de las propuestas funcionalistas sobre el particular (Renfrew 1976b). La monumentalidad del megalito, su posición visible sobre el paisaje (Criado Boado 1984/85, Fleming 1972, Lynch 1975, RCHM 1970: 427, Vaquero Lastres 1988), su relación con límites naturales y ecológicos que más tarde han dado lugar a fronteras humanas y sociales (extremo frecuente dentro del megalitismo: Criado Boado et all. 1986, Ellison et all. 1972, Lynch 1975), son otros tantos datos en este sentido.

Si se considera la relación con el tiempo, el rasgo más notable que representa el megalitismo (y otros tipos de monumentalismo) es la completa transformación de la actitud hacia el mismo que aparece en el pensamiento mítico, pues mientras éste es un recurso para matar o suprimir el tiempo (Lévi-Strauss 1976: 548), el monumento que se hace perenne es una forma de recuperarlo. En este sentido el megalito es un eco arcano, pero próximo, de lo que más tarde serán los Museos, los libros, el Pasado, la Tradición y la Historia.

Esta inversión en el orden del tiempo, de indudable relevancia y alcance, es coherente con la "domesticación del espacio" que supone los inicios del mundo campesino. Dentro de éste, de un sistema basado en los ritmos reiterativos de las estaciones y las cosechas, el tiempo, el ciclo y la repetición se hacen importantes. El campesino, a diferencia del salvaje, es un hombre concernido directamente con el control y cómputo del tiempo (Clastres 1981b). Es más, lo significativo no es que se contraponga un ahora con tiempo frente a un antes sin él (propuesta que sería demasiado simplista), sino que el tiempo cambia de configuración: frente a un tiempo estancado en el que el futuro no existe y la diferencia entre presente y pasado se expresa "espacialmente", bajo la forma de dos territorios distintos y paralelos, ahora surge un tiempo lineal presidido por la sucesión (22).

Podemos enmarcar el tiempo megalítico dentro de estas coordenadas en función no sólo de las evidencias consideradas al inicio de este apartado, sino también del hecho de que los megalitos contienen formas más o menos rudimentarias de calendarios. Sin necesidad de entrar en una revalidación o crítica de los postulados que diferentes trabajos han propuesto en torno a la ciencia y la astronomía megalitica (Bernatt et all. 1986, Heggie 1981, Ruggles et all. 1981), o a su posible significación social (ver Thorpe 1981), nos llega con que la mayor parte de los megalitos estén orientados en función de algún tipo de evento astronómico (23), lo que supone una observación continua de todos los ciclos de ese evento.

Así pues, y a modo de observación general, podemos decir que el punto más relevante del megalitismo de acuerdo con el planteamiento de este artículo lo constituye la paradoja de que este fenómeno, rompiendo equilibrios previos, expresa la preminencia de la cultura sobre el espacio a través de recursos temporales. En este sentido, y en contra del orden primitivo-salvaje el megalitismo representa el inicio de la victoria del tiempo sobre el espacio, una victoria de la que todavía seguimos siendo esclavos (24).

(22) Véase como ejemplos de ellos: Males (1986) y Mamani (1986). Discutir toda la bibliografía en este sentido sería muy prolijo. Además de Clastres (1981b: 89-90), se pueden ver Curry y otros (1987a y 1987b), Giddens (1984: 200 y ss.), Leach (1954), Montmollin (1987), Shanks y otros (1987a y 1987c). Sinclair (1987).

(23) En Galicia la orientación más frecuente de las cámaras es hacia el solsticio de invierno.

(24) Nos referimos a las miserias que el énfasis en el tiempo y el discurso teleológico llevan siempre aparejadas. Ver Bermejo Barrera (1987: libro II), o Foucault (1979). 


\section{PERSPECTIVA ESPECIFICA: LOS ESPACIOS MEGALITICOS}

Habiendo llegado hasta aqui, una cosa está bastante clara: la aproximación que hemos elegido no explica casi nada sobre megalitos. Esto, lejos de ser un error, es algo inherente al planteamiento del trabajo, al hecho de entender a los megalitos como "acontecimiento de pensamienton. Así pues, después de haber hablado del megalitismo y del pensamiento, consideraremos ahora el nivel del acontecimiento y los megalitos. En este punto habrá que considerar los contextos concretos dentro de los cuales surgen los monumentos megaliticos. Ello planteará que, al contrario de lo que ocurría en el nivel anterior, no sólo será posible, sino sobre todo necesario, utilizar el concepto ideología, buscando dónde y cuando ésta aparece, mostrando cómo la interrelación de poder, ideologia y sociedad (25) dinamiza la estructura del sistema de pensamiento subyacente a los monumentos megalíticos y cómo todas esas estrategias se conjugan (o conjuran) para construir instrumentaciones especificas de ese pensamiento dentro de contextos históricos particulares. Para ello, además de los conceptos anteriores, será preciso utilizar las nociones de acontecimiento y regularidad.

\subsection{Más allá de la típología: acontecimientos y regularidades megalíticas}

A lo largo del apartado anterior hemos insistido en que el megalitismo es un fenómeno monumental. Sin embargo, la monumentalidad megalitica no se reduce a la aparatosidad de su arquitectura, sino que la construcción de la monumentalidad megalítica incorpora un amplio repertorio de elementos que comprenden desde la distribución de los túmulos en el entorno, hasta los propios ajuares, concebidos éstos como un recurso material para significar una especie de monumentalidad interior del megalitismo. De este modo, el «monumentalismon megalítico sería la consecuencia de la conjugación y articulación de una serie de espacios diversos que se escalonan desde el exterior del monumento hasta su interior y entre los cuales, a menudo, se establecen relaciones de oposición o tensión espacial.

La resolución de esas tensiones de una u otra forma, el predominio de unos y otros elementos, da lugar a soluciones megaliticas muy dispares. En terminología convencional cada una de esas soluciones sería considerada un "tipo" específico de megalito. Sin embargo rechazaremos esa notación por limitarse a insistir en aspectos formales. En cambio, nos interesa destacar que, entendiendo cada tipo como una solución estratégica y significativa dentro de un contexto específico de los frentes de conflicto que se generan entre los diferentes niveles espaciales que integran el megalitismo como fenómeno cultural unitario, se podría llegar a describir las circunstancias que determinan la utilización de una u otra solución megalítica y, de este modo, a descubrir el sentido que la construcción de un monumento concreto podría tener.

Tomando como caso de estudio el $N W$ peninsular, que es la zona que el presente autor mejor conoce, podemos ver que los niveles espaciales de monumentalidad que integra el megalitismo son: la distribución general de las mámoas, o escala de asentamiento (Bello Diéguez et all. 1985 y 1987, Criado Boado et all. 1986), su localización puntual, o escala de emplazamiento (Criado Boado 19845, Vaquero Lastres 1988), el túmulo, la cámara (Criado Boado et all. 1986: 79 y ss.) y el ajuar (Fábregas Valcarce et all. 1988).

Esos "espacios", por su parte, en vez de limitarse a actuar estáticamente dentro de cada megalito, se interaccionan entre ellos dando lugar a horizontes de conflictividad. Los más señeros de estos últimos son: la interrelación monumento-entorno, el equilibrio túmulo-cámara, la dialéctica

(25) Sobre los conceptos ideología y poder ver sobre todo Miller y Tilley (1984b), además de los trabajos contenidos en el volumen Miller y Tilley (1984a). Ver también la nota x. 
cámara-estructuras de entrada $y$, finalmente, la relacion del ajuar con el resto del monumento (presencia/ausencia, mayor/menor importancia del mismo).

En el megalitismo del NW peninsular, al igual que en el de cualquice otra zona geográfica, es posible diferenciar elementos dispares dentro de cada uno de los niveles espaciales o frentes de conflicto que se han enumerado (26). Por nuestra parte, denominaremos acontecimiento al hecho de que un elemento concreto entre toda la serie posible sea el adoptado para ser utilizado y satisfacer los requerimientos de un determinado nivel espacial. La ventaja de esta conceptualización estriba en que con ella, en vez de limitarnos a hablar de meros elementos formales, comprendemos el proceso complejo a través del cual un elemento concreto es adoptado, destacando al mismo tiempo su articulación con otros elementos, su significado y el contexto mismo que rodea su utilización.

Al mismo tiempo, a las soluciones generadas por la yuxtaposición de acontecimientos concretos, a los resultados de las posibles combinatorias espaciales, les denominaremos regularidad. Poco más arriba se indicó que esas soluciones componen "tipos" específicos de megalitos. Sin embargo, del mismo modo que acontecimiento no equivale a elemento formal, regularidad tampoco se puede identificar con momento tipológico. El empleo del concepto regularidad permite introducir referencias al significado y al contexto que no entran dentro de la normativa tipológica.

Más en general podemos decir que la utilización de estas categorías teóricas nos impide caer en la perspectiva tradicional que, cuando descubre la aparición de un nuevo elemento formal, lo mantiene dentro del cuadro de evolución tipológica de ahí en adelante. En cambio, de un modo semejante a como ocurría con la "genealogía del poder" (Foucault 1980c y 1984) lo que realmente importa respecto a cualquier elemento que se considere, no es su origen ni cuándo ocurre por primera vez, sino todas las veces que se repite y por la razón que sea, configurando de ese modo acontecimientos particulares, y cada acontecimiento un momento fuera del tiempo teleológico que es la "presentización" de un instante (27).

Sintetizando ahora todos los diferentes acontecimientos que se pueden dar dentro de cada uno de los niveles espaciales y horizontes de conflicto considerados hasta aquí, podemos definir varias regularidades megalíticas distintivas. Ahora bien, la discusión que sigue, en vez de ser tomada como la tipificación de tres regularidades concretas, debería ser entendida como la descripción general de tres grupos de regularidades. Lo que constituye una regularidad determinada es cada megalito individual: cada mámoa singular es un acontecimiento único que concreta en un momento perpétuo toda la monumentalidad del megalitismo utilizando para ello rasgos y elementos procedentes del acervo general de este fenómeno cultural.

En primer lugar tendríamos un tipo de monumentalidad megalítica que se construye fundamentalmente en torno al túmulo y a la situación de éste en el espacio circundante. Las dimensiones de la mámoa y el emplazamiento en zonas de dominio topográfico o de amplia visibilidad confieren al monumento ese carácter que se podría definir como de predominio de la monumentalidad exterior, esto es, de una monumentalidad hacia fuera. En cambio los elementos más interiores apenas son destacados. La cámara está encerrada dentro del túmulo, es de escaso desarrollo y no tiene estructuras de entrada o éstas son de poca entidad. Los ajuares finalmente son pobres o casi inexistentes.

En segundo lugar tendríamos otra regularidad en la que se percibe un predominio o inicio de hegemonía de los elementos interiores. Las cámaras aparecen destacando en la superficie del túmulo, generalmente están dotadas de vigorosos corredores, según los datos arqueológicos que se

(26) Desafortunadamente queda fuera de los limites del presente trabajo la posibilidad de of recer una descripción pormenorizada de lo que ocurre en cada uno de esos niveles. Un primer intento en este sentido, así como un boceto de las interacciones espaciales que a continuación se señalan, fue ofrecido en Criado Boado y Fábregas Valcarce (1986). Además de ello, aspectos materiales sobre el megalitismo del NW han sido sistematizados recientemente en Fábregas Valcarce (1988a, 1988 b y 1988c).

(27) Estos conceptos están tomados de Foucault (1979, 1980b) y Bermejo Barrera (1987), Sahlins (1985) ofrece una categorización con observaciones interesantes en esta linea; para él un evento (event) es la relación existente entre un acontecimiento de la vida cotidiana y la estructura subyacente; como tal permite «la realización práctica de las categorías culturales dentro de un contexto histórico específico" (Sahlins 1985). Todo ello nos aporta unas «herramientas" teóricas útiles para desenvolver la perspectiva adoptada por cuanto, a través de ellas, se dinamiza la situación descrita anteriormente a nivel del pensamiento, y que, por imperiosidades metodológicas, podría parecer inmovilista y estática. En este sentido esas nociones son las más adecuadas para introducir las consideraciones planteadas más arriba sobre el pensamiento como «vocabulario». Ver también Criado Boado (1989 y A). 
han empezado a recuperar en los últimos años aparecen atrios y umbrales de considerable importancia (Criado Boado et all. 1986. Fábregas Valcarce et all. A), y los ajuares se hacen más abundantes y "ricos». Es relativamente frecuente la aparición en estos monumentos de elementos de cultura material de muy posible procedencia exterior.

Sin embargo, se debe anotar inmediatamente que lo que aqui denominamos elementos interiores no constituyen un grupo homogéneo, sino que dentro de esos elementos interiores se descubren elementos dispares e incluso contrapuestos. De este modo es posible diferenciar regularidades específicas dentro de este grupo genérico. Las dos básicas serian: la que se establece cuando los elementos interiores se inclinan del lado de los elementos claramente internos, esto es, cuando se asienta el predominio del espacio de la cámara, y la que, por el lado contrario, se genera cuando dentro de los elementos interiores priman los que se refieren a la transición entre interior y exterior, esto es, cuando se privilegia el atrio o la deposición del ajuar en el umbral.

En cualquier caso, lo que nos parece representativo del presente grupo de regularidades es que, en contraposición con esta hegemonía de los espacios interiores, los elementos exteriores disminuyen su importancia: el túmulo reduce su tamaño, llegando incluso cámaras que miden 6 ú $8 \mathrm{~m}$. de largo a estar encerradas por tumulos de 15 a $18 \mathrm{~m}$. de diámetro. E incluso es frecuente que ocupen posiciones topográficas deprimidas en las cuales, si bien aún poseen un emplazamiento elegido en función de determinadas condiciones de visibilidad (es el caso de A Arca de Barbanza: Criado Boado 1984-5), han desaparecido las exigencias de emplazamiento que se encuentran en otros monumentos.

De acuerdo con la evidencia de la distribución de túmulos, es posible que grupos que construian monumentos de este estilo, y tal vez en momentos avanzados del megalitismo, se hayan extendido hacia tierras bajas y hacia entornos ecológicos diferentes a los más característicos del momento megalítico del paisaje gallego.

Otra regularidad distinta estaría señalada por un grupo de mámoas que parecen representar el final de la tradición megalítica. En ellas la tendencia anterior y clásica, si se quiere, del megalitismo se interrumpe y cambia radicalmente de sentido. La forma más apropiada de definir esta regularidad tal vez fuese decir que en ella no es importante ni el espacio exterior ni el interior. Sólo el ajuar se hace importante. Este se convierte en el aspecto más distintivo de los monumentos de este estilo, por cuanto en ellos aparecen con frecuencia cerámica campaniforme, objetos metálicos, joyas incluso de oro... en suma todo el elenco de piezas consideradas por la bibliografía tradicionalmente como útiles de prestigio (Criado Boado y Vázquez Varela 1982, Fábregas Valcarce 1983, La Iglesia 1909, Maciñeira 1941, Rodríguez Casal 1983, Vázquez Varela 1979).

En cambio los monumentos se vuelven invisibles casi a todos los niveles, pues por un lado los túmulos decrecen de tamaño hasta casi desaparecer, pierden todo rastro de prominencia sobre el paisaje y, finalmente, la cámara, cuando la hay, pues son asimismo frecuentes las estructuras tumulares de tierra limpia y sin resto de cámara, reduce su tamaño y proporciones hasta la escala de una cista megalítica y queda englobada dentro del túmulo.

Esta propuesta de regularidades se basa, de hecho, en modelos o síntesis generales que han sido presentadas y sostenidas por diferentes autores. Se puede observar que, grosso modo, el esquema que se ha planteado reproduce los elementos básicos de las secuencias crono-tipológico-culturales elaboradas para dar cuenta tanto del megalitismo gallego (28), como incluso del N. de Portugal (29).

(28) Véanse los modelos tripartitos descritos por Rodríguez Casal (s. f.: 219), Vázquez Varela (1979: 24), VV.AA. (1979: 26).

(29) En esta zona se ha propuesto que: «De qualquier forma, uma evoluçao apartir de dólmens pequeños, simples, até formas de maiores dimensoes, com corredor destacado, ou já indiferenciado, dando depois progressivamente lugar a soluçoes de novo mais pequenas, mais «disfraçadas" na paisagem, que culminan nas pequeñas cistas e em sepulturas planas completamente invisiveis á superficie, da Idade do Bronze adiantada, apesar de ser uma ideia certamente simplista, continua a ser a que mais se adequa aos fragmentários dados existentes, desde que corrigida pela provável contemporaneidade de formas diferentes, nas mesmas necrópoles» (Jorge, 1984: 270). A continuación este mismo autor sostiene la validez de un esquema general de evolución de lo simple a lo complejo en la dinámica de la arquitectura megalítica de esta zona portuguesa, aunque advirtiendo que, además de no ser posible ofrecer evolución cronológica con pautas fijas (Jorge, 1984: 264), tamaña «tendencia evolutiva geral» no contradice la pervivencia de tipos antiguos y su existencia simultánea al lado de formas más evolucionadas (Jorge, 1984: 271). En este sentido se manifiesta este mismo autor en un trabajo distinto hablando en favor del polimorfismo arquitectónico y de la pervivencia de formas dentro del megalitismo de esa área (Jorge, 1986). 
Ahora bien, una de las diferencias básicas que distancia a nuestro esquema de esos otros, radica en el hecho de que con el no pretendemos establecer un esquema cronológico ni una secuencia tipológica o cultural. Esta posición es la que nos confiere un planteamiento genealógico (30), en el que el énfasis en el acontecimiento, la regularidad y la serie es más importante que la búsqueda de los orígenes, nacimientos y evoluciones de elementos o tipologias determinadas. Desde este punto de vista, para nosotros lo significativo es que en unas zonas convivan en el mismo momento diferentes combinaciones, o que mientras en ellas se mantiene una regularidad especifica, en otras áreas se adopten soluciones ya no desemejantes, sino incluso radicalmente opuestas, 0 , en definitiva, que comparando dos zonas dadas se pueda observar que la serie de regularidades que se percibe en cada una de ellas discurra en sentidos contrarios.

¿Cuál sería entonces la racionalidad que opera debajo de la elección de uno $u$ otro tipo de regularidad? Para ensayar una posible contestación a esta pregunta debemos, nuevamente, ensanchar el campo de observación y dirigirnos hacia el siguiente apartado.

\subsection{Estrategias de poder y anti-poder: las soluciones megalíticas}

A efectos de plantear una lectura imaginaria, vamos a sintetizar la síntesis que se ofreció en el apartado anterior. Tal y como se pudo observar, todos los acontecimientos y regularidades que se han definido se reducen o articulan alrededor del enfrentamiento exterior / interior como forma de construir la monumentalidad megalítica, circunstancia por otra parte normal cuando el fenómeno involucrado es de tipo religioso, funerario y simbólico, esto es, un tipo de fenómenos que basan su propia condición de existencia en el establecimiento de una clara distinción entre lo introducido en su lógica y lo exterior, periférico, marginal o profano.

A partir de esa oposición radical se configuran diferentes alternativas que expresan el sentido del megalitismo para comunidades particulares, y que se construyen utilizando unos elementos u otros para significar y concretar dicha oposición. Esos elementos, grosso modo, se escalonan a lo largo de una serie que posee tres escalas principales: énfasis en la monumentalidad exterior (en el túmulo y/o en el emplazamiento, por lo tanto), énfasis en la monumentalidad interior (en la cámara y/o en el ajuar), énfasis en el umbral (concretada en atrios y/o en ajuar depositado fuera de la cámara). La utilización de cada uno de estos elementos es para nosotros un acontecimiento, ya que señala la actualización de un recurso formal en un contexto específico. Y la suma de esos acontecimientos que dan lugar a un megalito, configuran un instante megalitico. Los tres, de hecho cuatro, (grupos de) instantes y regularidades megalíticas fundamentales que nos han sido dado ordenar, se corresponden con cada uno de esos tres niveles anteriores.

Ahora bien, se puede observar, sin ánimo de hacer una aproximación exhaustiva ni excesivamente general, que las lineas básicas que hemos caracterizado en el megalitismo gallego, se encuentran en la mayor parte de las "provincias" del megalitismo Atlántico. El mismo esquema de evolución dual del megalitismo europeo propuesto por Renfrew (1983b, 1984b y 1987a) responde a esa genealogía.

En Inglaterra, Fleming (1973) descubre una dinámica de dirección opuesta en el desarrollo de la arquitectura megalítica que, por un lado, pondría el énfasis en la función monumental a través del túmulo, y por otro en la función funeraria a través de la cámara. Las líneas de fuerza del megalitismo de Gran Bretaña discurren en torno a ese eje, dando lugar en unos casos a la hegemonía de las cámaras, en otros a la de los túmulos y en otros, a través de unas soluciones que los autores clásicos interpretan como de consenso o equilibrio entre las dos tendencias anteriores, a las grandes fachadas monumentales que caracterizan un importante grupo de túmulos megalíticos

(30) Concepto tomado de M. Foucault (1979 y 1980c): aplicado arqueólogicamente en el sentido de Criado Boado (1988b). 
británicos (31). También en Irlanda se percibe esta lógica de desarrollo de la arquitectura megalitica (32).

Los trabajos de C. Tilley en el sur de Suecia son también muv indicativos. Este autor considera la transición de la cultura TRB del neolitico Medio a la BAC (Battle Axe-Corded Wared Tradition) del neolitico Final, haciendo una descripción de la misma en términos ideológicos y simbólicos (Tilley 1984, Shanks y Tilley 1987a). Después de establecer una estrecha correspondencia entre la evolución de la decoración cerámica de la TRB y BAC, y el tipo de distribución de la primera dentro de los monumentos megalíticos y la correlación de la segunda con un nuevo tipo de monumento funerario, se vincula esa dinámica en la cultura material con un cambio en los principios simbólicos de ordenación del espacio que utilizaban esas comunidades, relacionando todo ello además con las circunstancias económicas y sociales de esos grupos.

De este modo se establece que, dentro de los grupos TRB (que construían monumentos megalíticos en cuyo interior eran depositados los muertos sin ajuar, después de haber anulado su individualidad a través de la práctica de la descarnación o la quema de sus cuerpos), y dentro de los cuatro periodos en los que tradicionalmente se divide esa tradición cultural, se pueden diferenciar cuatro estilos cerámicos totalmente dispares que se corresponden con cuatro pautas diferentes de deposición del ajuar en las tumbas. Así, en el periodo I y IV aparecen tipos cerámicos con escasa decoración, lo que, para el primer momento, es interpretado por el autor como reflejo de un énfasis escaso en destacar la identidad de los grupos particulares; durante estos momentos apenas se depositan ofrendas ni en el exterior, ni en el interior de la tumba. En cambio, en los períodos II y III se produce un incremento de la complejidad decorativa que se corresponde con el auge de la deposición de ofrendas en el exterior del monumento, destacando, posiblemente, a través de ello el incremento de las actividades y funciones rituales del megalito. En este último sentido, los autores interpretan que mientras las fases I y IV habrian estado polarizadas hacia lo interior, las fases II y III lo habrían estado hacia el exterior (Shanks y Tilley 1987a: 155-71).

La dinámica que se percibe en la fase IV, se continúa e intensifica con la tradición BAC, no sólo a nivel de decoración cerámica (ver Tilley 1984: fig. 19), sino sobre todo a nivel de ritos funerarios: en este momento se produce un abandono de la construcción de monumentos megalíticos, que son sustituidos por inhumaciones en sepulturas planas que ya no son "highly visible landmarks", y en las que el rito de enterramiento es la deposición individual de cuerpo entero. En algunos casos estas comunidades siguen utilizando los megalitos, pero depositando sus muertos con el rito de la nueva época y acompañados de los ajuares que caracterizan a estas comunidades, constituidos por hachas de combate y bipennes que son interpretados tradicionalmente como signo de intensificación de la jerarquización social (33).

Otros estudios realizados en la zona del Rhin y en la llanura Centroeuropea señalan también una dinámica de este estilo (Hodder 1982c, 1984).

Desde nuestro punto de vista, el interés de estas recurrencias y tendencias generales dentro del megalitismo atlántico radica en que, con independencia de las soluciones locales que se encuentren, permiten identificar unas líneas de fuerza básicas dentro de ese fenómeno cultural. De este modo, lo general y universal dentro del megalitismo no sería la presencia de esos procesos u otros cualquiera,

(31) Britnell (1979: 132-4), Corcoran (1969: 95-100), Darvill (1982), Henshall (1978), Lynch (1972), Manby (1976: 148). Savory (1980): ver en general Masters (1983). En algunos casos se rastrea una posible secuencia cronológica para la adquisición y pedominio de una u otra solución; de todos modos este tema es punto de debate entre los diferentes autores. Así se ha señalado en Escocia (ver Corcoran 1972, Henshall 1974, Scott 1969).

(32) En este caso los momentos señeros están representados por tumbas reducidas, con cámaras pequeñas encerradas en ellas y ajuares pobres, y en el polo opuesto, grandes monumentos tipo New Grange: ocupando una posición intermedia aparecen las tumbas de corredor, los portal tombs y los court tomsbs dotados de aparatosas estructuras de fachada y atrio (O'Kelly 1983). De acuerdo con los datos obtenidos en las excavaciones de la necrópolis de Carrowmore, es casi seguro que esa secuencia tipológica se corresponde asimismo con periodización cronológica (Burenhult 1984: 139-40).

(33) El autor que seguimos prefiere entenderlos únicamente como símbolo de un mayor auge en la individualización de los miembros del grupo, que también se realizaría a través del cambio en los ritos de enterramiento; este individualismo no excluye el incremento de la jerarquización y de las diferenciaciones sociales, ya que incluso sería posible que se desarrollara como complemento de este tipo de procesos; pero ofrece un marco menos restrictivo para entender todas las implicaciones del cambio de ritual funerario y de tipo de cultura material. 
sino la existencia en su base de unas oposiciones significativas que contienen las condiciones de posibilidad para generar un sentido que cada actualización concreta del megalitismo terminará de construir dentro de contextos concretos. Aún a riesgo de parecer aportar una explicación mecanicista, por tratarla con escaso detalle, intentaremos delimitar las lineas generales de estos procesos.

En el apartado anterior vimos que uno de los rasgos básicos del megalitismo es hacer visible la muerte. Sin embargo, este hecho es paradójico, pues al mismo tiempo que el megalitismo exhibe la muerte a nivel de todo el grupo, la oculta. Como fenómeno de enterramiento colectivo que es, supone un enmascaramiento del muerto individual. Esto se revigoriza con prácticas como la descarnación o la posible deposición secundaria en los megalitos, que, como se ha señalado en repetidos casos, tienen por objetivo negar la identidad individual en la muerte, (ver respectivamente Shanks y Tilley 1982 y 1987a, y Bloch 1971, Fraser 1983 y Hertz 1960). También encaja con esto la escasa importancia del ajuar y el hecho de que las piezas recuperadas en los megalitos representen, más que "ajuares individuales" propiamente dichos, of rendas de tipo general o restos de actividad ritual.

A la vista de esta situación contradictoria, en la que exhibición y monumentalismo funerario conviven con ocultación y desindividualización, y teniendo en cuenta las descripciones consideradas al hablar de la muerte en las SP, resulta tentador proponer que el megalitismo representa una fase de transición paulatina en la que se empezaría realizando una primera utilización de la muerte y recuperación del tiempo a nivel de todo el grupo, pero sin descender todavía al nivel individual.

Esta interpretación podría venir apoyada por el hecho de que cada una de las "regularidades" consideradas aqui, puede ser definida como una escala distinta en la intensidad de exhibición de la muerte. Así, la primera regularidad, en la que existe una invisibilidad de los ajuares y la cámara, se correspondería con un énfasis mayor en los aspectos espaciales y temporales del megalitismo, que en los funerarios. La segunda regularidad, en cambio, marcaría una polarización de ese énfasis hacia la dimensión ritual e interactiva del monumento: el individuo se sigue ocultando, pero la cámara que lo encierra adquiere mayor preminencia y, sobre todo, se hacen importantes los umbrales y espacios de actividad social, en los cuales se centran los restos materiales, en vez de en los ajuares individuales de los muertos.

Cada una de estas situaciones puede reflejar, en ese extraño conflicto entre la hegemonía espacial o la hegemonía temporal y funeraria, a través de esa contraposición que parece reproducir la pugna entre la sociedad y el individuo y, tal vez, entre la sociedad indivisa y la sociedad dividida, la expresión ideológica particular de una determinada estrategia de poder.

La situación se resuelve finalmente con la tercera regularidad, donde existe un énfasis absoluto en la exhibición del individuo, plasmada en la "privatización" y enriquecimiento de los ajuares, en el cambio a un rito de enterramiento individualizado y en la aparición de la inhumación. No es coincidencia entonces que la definitiva individualización en la muerte acompañe a la instauración de una nítida jerarquización social (34).

Claro que también se podría dar otra explicación. Pues de la misma forma que Clastres (1976b), plantea acerca de los potlach y de algunas sociedades de "grandes hombres" cabría la posibilidad-de que los megalitos, en vez de ser la expresión de los primeros atisbos de división dentro de una sociedad, y su historia la Historia de la emergencia paulatina del poder, fuesen los últimos esfuerzos de algunas sociedades, conscientes ya de la posibilidad y riesgo de llegar a la división, por conjurar ese fantasma y permanecer indivisa.

La construcción de monumentos megalíticos sería entonces la curiosa versión de un potlach, una operación de proporciones descomunales que implica el consumo en beneficio del colectivo de los excedentes producidos y acumulados por la sociedad y/o por determinados sectores o individuos dentro de ella. El hecho de que esa función la satisfagan los monumentos megalíticos, esto es, construcciones de finalidad funeraria, estaría vinculado a las inquietudes y objetivos de una sociedad

(34) Aunque todo esto se infiere a través de la evidencia gallega que se ha considerado, se observa mejor en otras zonas, algunas de las cuales hemos citado aquí. Consideraciones de este tipo surgen a partir de los análisis de Shennan (1982) y sobre todo, Braithwaite (1984). 
que, habiendo fundamentado su supervivencia en el dominio del entorno, precisa mostrar su hegemonía sobre el tiempo y reivindicar su territorio apelando a la tradición y a sus muertos.

Las disparidades y contradicciones entre los diferentes acontecimientos megaliticos narrarian entonces las vicisitudes de cada una de esas historias particulares; pues mientras en unos casos el discurso de todo el grupo se las arreglaría para tener éxito y el poder dividido quedaría conjurado, en otros en cambio las primeras formas de poder dividido cuajarían en una ideología expresada a través de los megalitos para con-vencer al grupo.

Las dos descripciones que hemos aportado, se resumen en definitiva entendiendo al megalito como discurso contra el poder (dividido) o a favor de ese poder.

\section{PERSPECTIVA FINAL: EL «ESPACIO» DE ESTE ARTICULO}

Llega el momento de distanciarnos un poco de las conclusiones de este texto, lo que significa adentrarnos todavía más en él. Pues al fin y al cabo la coherencia con el planteamiento asumido, nos obliga a reconocer que, tal y como advertíamos en el párrafo introductorio, no nos podemos abrogar el privilegio de creer que nuestra descripción sea la culminación de nada. Antes bien, es necesario hacer de ella un nuevo objeto de la labor crítica, lo que supone introducir un tercer nivel de lectura en el texto a través del cual, del mismo modo que antes hicimos con otras interpretaciones sobre el megalitismo, se intentase descubrir cuál podría ser el discurso oculto que habla nuestro discurso, y cuál el contexto que le da sentido.

Así, desde una perspectiva final y actual es bastante evidente que toda la descripción anterior puede ser no más que el redoblamiento de una critica a nuestro sistema económico-social. En ella los «salvajes" serían utilizados como los depositarios de unos valores (ecológicos y primitivos) que nuestra cultura ha extraviado. Estariamos, en cierto sentido, reproduciendo el mito roussoniano del "buen salvaje" (35), amparándonos para ello en uno de los principales problemas de este trabajo: la descomposición paulatina de las sociedades primitivas, ya sea por aculturación o destrucción, no nos permite conocer auténticos salvajes. Ni tan siquiera sabemos si éstos existieron en algún momento o cómo fueron. Mientras tanto, la reflexión occidental se inventa una definición de lo salvaje para, de hecho, satisfacer un objetivo más oculto: definirse (y legitimarse) a sí misma (36).

Se podría optar por este último nivel de lectura o de credibilidad del presente texto. Pero también se debe valorar que la descripción anterior, en la medida en que sigue una ordenación rigurosa desde lo más general, y si se nos apura evidente, a lo más particular, y por lo tanto inconcreto, ofrece asimismo una graduación de niveles de credibilidad. El primer apartado aporta un esquema genérico (teórico y práctico) que se puede aplicar grosso modo a la prehistoria. El segundo plantea recursos teórico-metodológicos específicos y conclusiones particulares sobre el megalitismo cuya validez dependen exclusivamente de contextos locales. Posiblemente sea factible aceptar el texto anterior por etapas, en vez de en bloque.

En este sentido, y para finalizar, diremos que, sin haberlo buscado a propósito, nos ha salido un título simétrico y opuesto al de Renfrew (1976b). Evidentemente el término megalitos coincide en ambos títulos, pues no en vano constituye el objetivo (o pretexto) de ambos trabajos. En cambio, la oposición que se manifiesta entre los otros dos términos de cada título, señala con claridad la dispararidad de planteamiento y alcance de un trabajo y otro. Así, a «territorios" nosotros oponemos "espacio", ya que mientras el primer término define una concepción material e incluso burguesa del

(35) Una crítica de este tipo respecto a Lévi-Strauss aparece esbozada en Derrida (1976).

(36) Véanse los comentarios en este sentido de Duvignaud (1977: 227 y ss.). Asimismo es muy indicativo que, a menudo, diversos intentos de caracterizar o criticar nuestro sistema social, involucren la consideración de lo primitivo (Braudillard 1980: 71 y ss., Lyotard 1987: 35 y ss.) 
entorno (37), el segundo nos lleva al "paisaje», concepto que sintetiza la dimension física del medio natural con la dimensión simbólica del paisaje humano. Igualmente, al término ecologicista "poblaciones" oponemos "pensamiento" pues en tanto lo primero reduce al hombre a su proyección animal (38), lo segundo lo introduce dentro de sus componentes culturales.

Hemos tocado muchos temas dispares. Otros han sido únicamente sugeridos. La estrechez de espacio con la que nos movimos no nos permitió extendernos en discusiones más pormenorizadas. Como consecuencia de todo ello, algunas de las descripciones e interpretaciones aqui sugeridas es posible que parezcan inconexas, improcedentes o innecesarias. Al menos esperamos haber apuntado los contornos generales de una línea de búsqueda que nos parece fructífera.

\section{BIBLIOGRAFIA}

ADLER, (1980): Fazedores de chuva, fazedores de ordem. Reflexao sobre as relaçoes da magia e da poder na Africa Negra. En VV. AA 1980. (Libre 1, 1977); 91-115.

Althusser, L. (1985): Curso de filosofia para cientificos. Barcelona: Planeta-De Agostini, (Philosophie et philosophie spontanée des savants, Maspero, Paris, 1974); $159 \mathrm{pp}$.

BARNATT, J. y HERRING, P. (1986): Stone circles and megalithic geometry: an experiment to test alternative design practices. Journal of Archaelogical Science, 13, 5: 431-50.

Bello Diéguez, J. M. Criado Boado, F. y Vázouez Varela, J. M. (1985): Cultura megalítica y medio natural en el NW peninsular. Arqueologia Espacial. Coloquio sobre distribución y relaciones entre los asentamientos, VI, (Teruel); 229-35.

- (1987): La cultura megalitica de la provincia de la Coruña y sus relaciones con el marco natural: implicaciones socio-económicas. Diputación Provincial, Coruña; 163 pp.

Bermejo Barrera, J. C. (1983): Psicoanálisis del conocimiento histórico. Akal, Madrid; 181 pp.

- (1987): El final de la Historia. Ensayos de Historia Teórica. Akal, Madrid; 286 pp.

BLOCH, M. (1971): Placing the dead. Tombs, ancestral villages and kinship organisation in Madagascar. Seminar Press, London; 378 pp.

BOSERUP, E. (1965): Conditions of Agriculture Growth. Aldine, Chicago; 267 pp.

BOWEN, H. C. y FoWLER, P. J. (ed.). (1978): Early land allotment in the British Isles. A survey of recent work. Oxford: B.A.R. British Series, 48; 202 pp.

BRADley R. y GARDINER, J (eds.). (1984): Neolithic studies. A review of some current research. Oxford: B.A.R. British Series, 133; 218 pp.

BRAITHWAITE, M. (1984): Ritual and prestige in the prehistory of Wessex c. 2200-1400 BC: a new dimension to the archaeological evidence. En D. Miller y C. Tilley (eds.). 1984a; 93-110.

BRAudillaRD, J: (1978): Cultura y simulacro. Kairós, Barcelona. (Editions Galilée, Paris 1978); 99 pp.

- (1980): El espejo de la producción. Gedisa, Barcelona. (Le miroir de la production. Casterman, Paris. 1973); $178 \mathrm{pp}$.

BRITNELL, (1979): pp.

BRoSE, D. S. y GREBER, N. (ed.). (1979): Hopewell Archaeology: The Chillicothe Conference. Kent State University, Kent; 309 pp.

BURENHULT, G. (1984): The archaeology of Carrowmore: environmental archaeology and the megalithic tradition at Carrowmore, Co. Sligo, Ireland. Institute of Archaeology, Stockholm; 397 pp.

Carmichael, E., Hugh-Jones, S., Moser, B. y Tayler, D. (1986): The hidden peoples of the Amazon. British Museum, London; 96 pp.

CAULFIELD, S. (1978): Neolithic fields: the Irish evidence. En H. C. Bowen y P. J. Fowler (eds.). 1978; 137-43.

- (1983): The neolithic settlement of north Connaught. En T. Reeves-Smith y F. Hamond (eds.), 1983; 195-215.

- (1986): The structured landscape of tombs builders in the Irish neolithic, World Archaeological Congress, "The Neolithic of Europe" (Southampton, 1986).

(37) Estas implicaciones de la reflexión geográfica más tradicional sobre el espacio se pueden documentar, por ejemplo, en Dollfus (1975: 8); una crítica de estos planteamientos en Quaini (1981).

(38) Véase en Kormondy (1978: 91 y ss.) el concepto ecológico de población, Las reminiscencias ecologicistas de este concepto dentro de la Antropologia se pueden observar en Martínez Veiga (1978: 117 y ss.). 
Cl.ARKE, D. L: (ed.). (1972): Models in Archaeology. Methuen and Co. L.d., London; 1055 pp.

Clastres, P. (1975): A sociedade contra o Estado. Ediçoes Afrontamento, Porto. (Minuit, Paris. 1974); 213 pp.

- (1976)a: La cuestión del poder en las sociedades primitivas. En P. Clastres 1981a (Interrogations 7, 1976); pp. 109-16.

- (1976)b: La economia primitiva. En P. Clastres 1981a (prólogo a la edición francesa de M. Sahlins, Age de pierre, Age d'abondance, Gallimard, Paris. 1976); 133-51.

- (1977): El retorno de las luces. En Clastres 1981a (Revue franc. de science politique 1, Paris); 153-63.

- (1978): Los marxistas y su antropologia. In P. Clastres 1981a (Libre 3, Paris); pp. 165-79.

- (1981)a: Investigaciones en Antropologia Politica. Gedisa, Barcelona. (Recherches d'anthropologie politique, Seuil, Paris. 1980); 256 pp.

- (1981)b: Mitos y ritos de los indios de América del Sur. En P. Clastres 1981a (Dictionnaire des mythologies et des religions, Flammarion, Paris); 65-107.

- (1986): Crónica de los indios Guayakis. Lo que saben los Aché, cazadoras nómadas del Paraguay. Alta Fulla, Barcelona. (Plon, Paris. 1972); 252 pp.

COHEN, N. (1981): La crisis alimentaria en la prehistoria. Alianza Editorial, Madrid. (Yale, 1977); 327 pp.

Corcoran, J. X. W. P. (1969): The Cotswold-Severn Group. En T. G. E. Powell (ed.) 1969; 13-104.

- (1972): Multi-period construction and the origins of the chambered long cairn in Western Britain and Ireland. En F. Lynch y C. Burgess (eds.), 1972; 31-64.

CRIADO BoAdo, F. A. «We, the post-megalithic people...* En Hodder (ed.), The meaning of symbols. En prensa.

- B. Walking about Lévi-Strauss: is a structural archaeology still possible? En Hodder et al. (eds.) A; en prensa.

- (1984-5): «El tercer factor» o la lógica oculta del emplazamiento de los túmulos megalíticos gallegos. Cuadernos de Estudios Gallegos, XXXV.

- (1988)a: La genealogía del Paisaje: hacia una aproximación no funcionalista al estudio de las relaciones cultura-entorno. Ind Deya Conference.

- (1988)b: Arqueologia del Paisaje en Galicia. Arqueologia Espacial, 13, Teruel.

- (1989): Contribución al estudio de las relaciones entre las comunidades megaliticas del noroeste peninsular y su medio natural implicaciones socio-económicas. Universidad de Santiago: tésis doctoral presentada en la Fac. de Xeografía e Historia.

Criado Boado, F., Aira Rodríguez, M. J. y Diaz-Fierros VI-Queira, F. (1986): La construcción del paisaje. Megalitismo y Ecología en la Sierra de Barbanza. Xunta de Galicia, Dirección Xeral do Patrimonio Artístico e Monumental, Santiago; 185 pp.

Criado Boado, F. y Fábregas Valcarce, R. (1986): The Megalithic culture of northwestern Iberia, World Archaeological Congress, "The Neolithic of Europe" (Southampton, 1986).

Criado Boado, F. y Vázouez Varela (1986): La cerámica campaniforme en Galicia. Ediciós do Castro, Coruña; $104 \mathrm{pp}$.

Curry, P. y Montmoluin, O. (ed.). (1987)a: Archaeology and Time. Archaeological review from Cambridge, 6.1.

- (1987)b: Archaeology and Time. En P. Curry y O. Montmollin (eds.) 1987a; 2-4.

DARVILL, T. C. (1979): Court cairns, passage graves and social change in Ireland. Man, XIV: $311-27$.

- (1982): The Megalithic Chambered Tombs of the Cotswold-Severn Region. Vorda Publications, Highworth (Wiltshire); 149 pp.

DAveY, P. (ed.). (1978): Man and environment in the Isle of Man. B.A.R. British Series, 54(i); Oxford; 176 pp.

DAvidSon, D. A. y Shackley, N. L. (ed.). (1976); Geoarchaeology. Duckworth, London.

DAviES, P. C. W. (1982): El espacio y el tiempo en el universo contemporáneo. Fondo de Cultura Económica, México. (Space and time in the modern universe, Cambridge University Press, Cambridge. 1977); 399 pp.

DAY, G. M. (1953): The Indian as an ecological factor in the northeastern forest. Ecology, 34: 2: 329-46.

DERrIDA, J. (1976): Of Grammatologie. Johns Hopkins University Press, Balimore, Maryland. (De la Grammatologie, Paris, 1967); 354 pp.

Dollfus, O. (1976): El espacio geográfico. Oikos-Tau, Barcelona. (L'espace geographique, Presses Universitaires de France, Paris. 1976); 124 pp.

DuBy, G. (1980): Los tres órdenes o lo imaginario del feudalismo. Petrel, Madrid. (Les trois ordres ou l'imaginaire du féudalisme, Gallimard, Paris. 1978); 464 pp.

Duvignaud, J. (1977): El lenguaje perdido. Ensayo sobre la diferencia antropológica. Siglo XXI, México. (Le langage perdu, Presses Universitaires de France, Paris. 1973); 247 pp.

EAgleton T. (1986): Against the Grain. Essays 1975-1985. Verso, New Left Books, London; 199 pp.

- (1987): Awakening from modernity. Times Literary Supplement, February 20, 1987; pp. 194.

Ellison, A. y HaRRIS, J. (1972): Settlement and land use in the prehistory and early history of southern England: a study based on locational models. En D. L. Clarke (ed.) 1972; 911-962.

Evans, J. G., Limbreby, S. y Cleere, H. (ed.). (1975): The effect of man on the landscape: the Highland Zone, Council for British Archaeology, London. Research Report 11; 129 pp. 
Fábregas Valcarce, R. (1983): Materiales liticos del Museo Provincial de Lugo. Precisiones sobre la fase final de la cultura megalitica del N. O. peninsular. Boletin do Museo Provincial de Lugo, 1; 31-42.

- (1988)a: Presentación: panorámica del fenómeno megalítico gallego. En R. Fábregas Valcarce y F. de la Fuente Andrés 1988; 1-8.

- (1988)b: Le phenomenon megalithique dans le NW du l'Espagne, L'Anthropologie, en prensa.

- (1988)c: Megalitismo da Galiza, Coloquio de Arqueologia do NW peninsular (Porto, setiembre de 1988); en prensa.

FÁbregas Valcarce, R. y Fuentes Andrés (1988): Aproximaciones a la cultura material del megalitismo gallego: la industria litica pulimentada y el material cerámico. Arqueohistórica, 2. Publicaciones de la Universidad de Santiago, Santiago; 296 pp.

- A. Memoria de las excavaciones en túmulos megalíticos en Os Campiños, Leiro, (Rianxo, A Coruña). En prensa.

FAwCETT, C. (1986): The politics of assimilation in Japanese archaeology. Archaeological Review from Cambridge, 5: 1: 43-57.

Fleming, A. (1971): Territorial patterns in Bronze Age Wessex, Proc. of Prehistoric Society, 37; $138-66$.

- (1972): Vision and design: Approaches to Ceremonial Monument Typology. Man, VII: 57-73.

- (1973): Tombs for the living. Man, VIII: 177-93.

FORD, R. I. (1979): Gathering and Gardening: Trends and Consequences of Hopewell Subsistence Strategies. En D. S. Brose y N. Greber (eds.) 1979; pp. 234-8.

Foucault, M. (1978)a: Las palabras y las cosas. Siglo XXI, México. (novena edic.; 1968 primera edición; Gallimard, Paris. 1966); 375 pp.

- (1979): La arqueología del saber. Siglo XXI, México. (sexta edición; 1970 primera edición; L'archeologie du savoir, Gallimard, Paris. 1969); 335 pp.

- (1980)a: Historia de la sexualidad. 1. La voluntad del saber. Siglo XXI, Madrid. (tercera edición; 1977 primera edición; Histoire de la sexualité. 1. La volonté de savoir, Gallimard, Paris. 1976); 194 pp.

- (1980)b; El orden del discurso. Tusquets, Barcelona. (segunda edición; 1973 primera edición, L'ordre du discours, 1970); 64 pp.

- (1980)c: Nietzsche, la genealogia, la historia. En M. Foucault, Microfísica del poder. La Piqueta, Madrid; 7-29.

- (1984): Vigilar y castigar. Siglo XXI, Madrid. (cuarta edición; 1978 primera edic.; Surveiller et punir, Gallimard, Paris. 1975); 314 pp.

Gauchet, M. (1980): A divida do sentido e as razoes do Estado. Política de Religao Primitiva. En VV. AA (ed.) 1980, (Libre 2, 1977); 49-89.

Giddens, A. (1979): Central Problems in Social Theory. Macmillan, London.

- The Constitution of Society. Outline of the Theory of Structuration. Polity Press, Cambridge. (paperback edit. 1986); $402 \mathrm{pp}$.

GODELIER, M. (1976)a: Antropologia y Biologia. Anagrama, Barcelona. (Revue Internationale des Sciences Sociales, XXVI, 4, 1974); 56 pp.

- (1976)b: Funcionalismo, Estructuralismo y Marxismo. Anagrama, Barcelona. (Paris, 1972 ); 71 pp.

- (1977)a: Teoria marxista de las sociedades precapitalistas. Edit. Laia. Barcelona. (Editions Sociales, Paris. 1970); 161 pp.

- (1977)b: Sobre el modo de producción asiático. Edic. Martínez Roca, Barcelona; 269 pp.

- (1984): L'idéel et le matériel. Fayard, Paris; 349 pp.

- (1986): La producción de Grandes Hombres. Poder y dominación masculina entre los Baruya de Nueva Guinea. Akal, Madrid. (Fayard, Paris. 1982); 288 pp.

HARRIS, M. (1982): El materialismo cultural. Alianza Editorial, Madrid. (Cultural Materialism, Random House, 1979); 399 pp.

HARrIS, O. (1986): The politics of the Past. Anthropology Today, 2, 6; 19-20.

HegGIE, D. C. (1981): Megalithic Science. Ancient Mathematics and Astronomy in Northwest Europe. Thames \& Hudson, London; 256 pp.

Henshall, A. S. (1974): Scottish Chambered Tombs and Long Mounds. En C. Renfrew (ed.) 1974; $137-64$.

- (1978): Manx Megaliths again: an attempt of Structural Analysis. En P. Davey (ed.) 1978; 171-6.

HerTZ, R. (1960): Contribution to the study of the collective representation of death. En Death and the Right Hand. Cohen \& West, Aberdeen. (Contribution á une étude sur la représentation collective de la mort, Année Sociologique, X, 1907); 178 pp.

HoDDER, I; (ed). (1982)a: Symbolic and structural Archaeology. Cambridge University Press, Cambridge; 188 pp.

- (1982)b: Theoretical Archaeology: a reactionary view. En Hodder (ed.) 1982a; 1-15.

- (1982)c: Sequences of structural change in the Dutch Neolithic. En I. Hodder (ed.) 1982a; 162-177.

- (1982)d: Symbols in action. Ethnoarchaeological studies of material culture. Cambridge University Press, Cambridge; 229 pp. 
- (1982)e: The Present Past. An introduction to anthropology for archaeologists. Batsford Ltd., London; 239 pp.

- (1984): Burials, houses, women and men in the European Neolithic. En D. Miller y C. Tilley (eds.) 1984a; 5167.

- (1986): Reading the Past. Current approaches to interpretation in archaeologv. Cambridge University Press, Cambridge; 194 pp.

- (1987): The Archaeologv of Contextual Meanings. Cambridge University Press, Cambridge; 144 pp.

- (1987): La Arqueologia en la era post-moderna. Trabajos de Prehistoria, 44: 11-26.

HODDER, I. (ed), A: The meaning of symbols. Allen and Unwin. London. Forthcoming.

Hodder, I., Isaac, G. y Hammond, N. (eds). (1980): Patterns of the Past. Cambridge University Press, Cambridge; $635 \mathrm{pp}$.

HODDER, I., et al. A: Reading Material Culture. Scientifical Blackwell, Oxford. (en prensa).

JENNESS, D.: The Indians of Canada National Museum of Canada, Otawa; 452 pp.

JONES, R. L. (1976): The activities of Mesolithic Mand: further palaeobotanical evidence from Northeast Yorkshire. En D. A. Davidson y N. L. Shackley (eds.) 1976; 355-67.

Jorge, V. O. (1982): Megalitismo do Norte de Portugal: o distrito do Porto. Os monumentos e a sua problemática no contexto europeu. Tésis doctoral, Fac. de Letras, Porto; 953 pp.

- (1984): Megalitismo do Norte de Portugal: novos elementos. III Seminario de Arqueología do NW Peninsular, (Guimåraes, 1982), Revista de Guimãraes, XCIV: 263-89.

- (1986): Polymorphisme des tumulus préhistoriques du Nord du Portugal: le cas d'Aboboreira. Bulletin de la Societé Prehistorique Française, 83: 177-82.

Kormondy, E. J. (1978): Conceptos de Ecologia, Alianza Editorial, Madrid. (1973, primera ed.; Concepts of Ecology, Prentice Hall, New Yersey. 1969); 248 pp.

LAET, S. J. de (ed.), (1976): Acculturation and Continuity in Atlantic Europe, Dissertations Archaeologicae Gandenses, $X V I$.

LA IGLESIA (1907): Prehistoria gallega. Notas para un catálogo. Almanaque Ferrolano para 1907.

LARSSON, M. (1985): The Early Neolithic Funnel-Beaker Culture in southwest Scania, Sweden. Social and Economic Change, 3000-2500 BC. B.A.R. International Series, 142, Oxford; 184 pp.

Leone, M., Potter, B. P., Jr. y Shackel, P. A. (1987): Toward a Critical Archaeology, Current Anthropology, $28,3$.

LEVI-STrauss, CL. (1963): The use of wild plants in tropical South America. En J. H. Steward (ed.) 1963; $465-86$.

- (1964): El pensamiento salvaje, Fondo de Cultura Económica, México. (tercera reimpresión, 1975; La pensé sauvage, Plon, Paris. 1962); 413 pp.

- (1965): El totemismo en la actualidad. Fondo de Cultura Económica, México. (primera reimpresión, 1971: Le Totémisme aujourd' hui, Presses Universitaires de France, Paris. 1962); 159 pp.

- (1972): Estructuralismo y ecologia. Anagrama, Barcelona. (Structuralism and Ecology, Barnard Alumnae, New York. 1972); 41 pp.

- (1973): Antropología Estructural Eudeba, Buenos Aires. (primera edición, 1968; Anthropologie Structurale, Plon, Paris. 1961); 371 pp.

- (1976): El hombre desnudo. Siglo XXI, México. (L'homme nu, Plon, Paris. 1971); 697 pp.

Lızot, J. (1978): Economie primitive et subsistance. Libre, 4; 69-113.

- Populaçao, recursos e guerra entre os Yanomami Crítica da Antropología Ecológica. En VV. AA 1980, (Libre 2, 1977); 161-97.

LYNCH, F. (1972): Portal dolmens in the Nevern Valley, Pembrokeshire. En F. Lynch y C. Burgess (eds.) $1972 ; 67-$ 83.

- (1975): The impact of landscape on prehistoric man. En J. G. Evans et all. (eds.) 1975; 124-7.

LYNCH, F. y BuRgess, C. (ed.). (1972): Prehistoric Man in Wales and the West. Bath; 368 pp.

LYOTARD, J. F. (1984): La condición postmoderna. Edic. Cátedra, Madrid. (Minuit, Paris. 1984); 119 pp.

- (1987): La postmodernidad, (explicada a los niños). Gedisa, Barcelona. (La Postmoderne expliqué aux enfants, Galilée, Paris. 1986); 123 pp.

MACIÑEIRA Y PARDo DE LAMA, F. (1941): El vaso campaniforme y condiciones de sus intactos yacimientos tumulares en la estación de Puentes. Atlantis, XVI: 356-69.

MAESTRE Alfonso, J. (1978): Medio ambiente y sociedad. Edit. Ayuso, Madrid; 245 pp.

MAles, A. (1986): Pasado y Presente de las sociedades indigenas de los Andes: Los Otavalos de ayer y de hoy. World Archaeological Congress. Indigenous Perceptions of Past (Southampton, 1986).

Mamani Condori, C. (1986): Historia y Prehistoria: ¿dónde nos encontramos los indios? World Archaeological Congress. Indigenous Perceptions of Past (Southampton, 1986).

Martínez VeigA, V. (1978): Antropología Ecológica. Edit. Adara, Coruña; 246 pp.

- (1985): Cultura y Adaptación. Cuadernos de Antropologia. Anthropos, Barcelona; 56 pp.

MASTERS, L. (1983): Chambered Tombs and Non-Megalithic Barrows in Britain. En C. Renfrew (ed.) $1983 \mathrm{a} ; 97$. 112 . 
MAY, F. (1986): Les sepultures prehistoriques. Editions du CNRS, Paris; 264 pp.

Mellars, P. (1975): Ungulate populations, economic patterns, and the Mesolithic landscape. En J. G. Evans et al. (eds.) $1975 ;$ 49-56.

Midgley, M. S. (1985): The Origin and Function of the Earthern Long Barrows of Northem Europe. B.A.R, International Series, Oxford. 259; 236 pp.

MilleR, D. (1982): Artefacts as products of human categorisation. En I. Hodder (ed.) 1982; 17-24.

- (1985): Artefacts as Categories. Cambridge University Press, Cambridge; 283 pp.

Miller, D. y Tilley, C. (eds.). (1984)a: Ideology, power and prehistorv. Cambridge University Press, Cambridge; $157 \mathrm{pp}$.

- (1984)b: Ideology, power and prehistory: an introduction. En Miller, D. y Tilley, C. (eds.) 1984a; 1-16.

Montmoluin, O. (1987): Temporal and social scales in Prehispanic Mesoamerica. Archaeological Review from Cambridge, 6: 1: 51-61.

O'Kelly, M. J. (1983): The Megalithic Tombs of Ireland. En C. Renfrew (ed.) 1983a; 113-126.

OLSEN, B. (1986): Norwegian archaeology and the people without (pre)history: or how to create a myth of a uniform past. Archaeological Review from Cambridge, 5: 1: 25-42.

PoWELL, T. G. E., (ed.). (1969): Megalithic Enquiries in the West of Britain. Liverpool; 396 pp.

QUAINI, M. (1981): La construcción de la geografia humana Oikos-Tau, Barcelona. (La construzione della Geografia humana, Nuova Italia, Firenze. 1981); 245 pp.

RADCliffe-Brown, A. R. (1986): Estructura y función en la sociedad primitiva. Planeta-De Agostini, Barcelona. (Routledge \& Kegan Paul, London: 1969); 23 pp.

Rchm (1970): Dorset. Royal Commission on Historical Monuments, England. II. S. W. Dorset. HMSO, London; 427 pp.

ReEVES-Smith, T. y Hamond, F. (eds). (1983): Landscape archaeology in Ireland. B.A.R. British Series, 116. Oxford; 389 pp.

Renfrew, C. (ed.). (1974): British Prehistory. A New Outline. Duckworth, London. (tercera reimpresión, 1980); 348 pp.

- (1976)a: Before Civilization. Penguin Books, Middlexes. (London, 1972); 320 pp.

- (1976)b: Megaliths, Territoires and Populations. En S. J. de Laet (ed.) 1976; 298-320.

RENFREW, C. (ed.). (1983)a: The Megalithic Monuments of Western Europe. Thames \& Hudson, London. (London, 1981); 127 pp.

- (1983)b: The Megalithic Monuments of Western Europe. En C. Renfrew (ed.) 1983a; 8-17.

- (1984)a: Approaches to Social Archaeology. Edinburgh University Press, Edinburgh; 429 pp.

- (1984)b: Monuments and the structure of pre-urban societies. En C. Renfrew 1984a; 156-64.

- (1987): El arqueólogo y su pensamiento. Una entrevista con C. Renfrew. Revista de Arqueologia, 78: 5-13.

Rodríguez CaSal, A. (1983): A Mámoa de Monte Campelos e o seu enterramente secundario. Novos datos encol do Megalítico Final galego. Boletín do Museo Provincial de Lugo, 1: 7-17.

- s.f. Voz «Megalítico». Gran Enciclopedia Gallega, XX, 215-220.

Ruggles, C. y Whittle, A., (eds). (1981)a: Astronomy and Society in Britain during the period $4000-1500$ BC. B.A.R. British Series, Oxford. 88; 342 pp.

- (1981)b: A critical examination of the megalithic lunar observatoires. En C. Ruggles y A. Whittle (eds.) 1981a; 153-21.

Sahlins, M. (1977): Economía de la Edad de Piedra. Akal, Madrid. (Aldine Pub. Co., Chicago. 1974 ); 340 pp.

- (1985): Islands of History. University of Chicago Press, Chicago; $253 \mathrm{pp}$.

SAvORY, R. N. (1980): The Neolithic in Wales. En J. A. Taylor (ed.) 1980; 207-31.

ScorT, J. G. (1969): The Clyde Cairns of Scotland. En T. G. E. Powell (ed.) 1969; pp. 175-221.

SERVICE, E. (1973): Los cazadores. Labor, Barcelona. (Prentice Hall, New Jersey); 136 pp.

Simmons, I. G. (1979): Late Mesolithic societies and the environment of the uplands. Bull. of Inst. of Archaeology, 16: 111-29.

SINCLAIR, A. (1987): Time and class: some aspects of time in 17th and 18th century England, Archaeological Review from Cambridge, 6: 1: 62-74.

Shanks, M. y TILLEY C. (1982): Ideology, simbolic power and ritual communication: a reinterpretation of Neolithic mortuary practices. En I. Hodder (ed.) 1982a; 129-53.

- (1987)a: Re-Constructing Archaeology. Cambridge University Press, Cambridge; 267 pp.

- (1987)b: Archaeology and Social Theory. Polity Press, Cambridge; 185 pp.

- (1987)c: Abstract Time and Substantial Time. Archaeological Review from Cambridge, 6: 1: 32-41.

ShEnNAN, S. (1982): Ideology, Change and the European Early Bronze Age. En I. Hodder (ed.) 1982a; 155-61.

Sherratt, A. (1980): Plough and Pastoralism: Aspects of the Secondary Products Revolution. En I. Hodder et al, (eds.) 1980; 261-305. 
SPERber, D. (1985): On Anthropological Knowledge. Cambridge University Press, Cambridge. (Le Savoir des Anthropologues, Hermann, Paris. 1982): $110 \mathrm{pp}$.

Steward, J. H., (ed.). (1963): Handbook of South American Indians, 6: Physical Anthropology, Linguistics and Cultural Geography of South American Indians. Cooper Square Pub. New York.

TAYlor, J. A. (ed.). (1980): Culture and environment in Prehistoric Wales. B.A.R. British Series, 76, Oxford; 387 pp.

THORPE, I. J. (1981): Ethnoastronomy: its patterns and archaeological implications. En C. Ruggles y A. Whittle (eds.) $1981 ; 275-88$.

- (1984): Ritual, power and ideology: a reconstruction of earlier neolithic rituals in Wessex. En R. Bradley y J. Gardiner (eds.) 1984: 41-60.

ThorPE, I. J. y RichaRDS, C. C. (1984): The decline of ritual authority and the introduction of beakers into Britain. En R. Bradley y J. Gardiner (eds.) 1984; 67-85.

Til.LEY, C. (1984): Ideology and the legitimation of power in the Middle Neolithic of Southern Sweden. En D. Miller y C. Tilley (eds.) 1984; 111-45.

TricarT, J. y Kilian, J.: (1982): La eco-geografia y la ordenación del medio natural Anagrama, Barcelona. (L'écogéographie et l'aménagement du milie naturel, Maspero, Paris. 1979); 288 pp.

VAOUERO LASTRES, X. (1988): ¿Dónde diablos están nuestros muertos que no se dejan ver? Brigantium, VI, en prensa.

VAтtimo, G. (1987): El fin de la Modernidad. Nihilismo y hermeneútica en la cultura postmoderna. Gedisa, Barcelona. (La fine della modernitá, Torino, 1985); 160 pp.

VÁzoufz Varfia, J. M. (1979): El horizonte de Rechaba. Una nueva fase de la cultura megalítica del Noroeste Peninsular. Boletin Auriense, IX: 9-26.

VV. AA. (1979): Prehistoria e Arqueoloxia de Galicia. Estado da cuestión. Instituto de Estudos Galegos Padre Sarmiento, Lugo; 78 pp.

- (1980): Guerra, religiao, poder. Ediçoes 70, Porto; 199 pp. 